\title{
50. COSTA RICA RIFT: VARIABLY DEPLETED BASALTS IN THE SAME HOLE1
}

\author{
J. Etoubleau, ${ }^{2}$ O. Corre ${ }^{2}$ J. L. Joron, ${ }^{3}$ H. Bougault, ${ }^{2}$ and M. Treuil ${ }^{4}$
}

\begin{abstract}
The basalts recovered from the Costa Rica Rift by drilling at Deep Sea Drilling Project Sites 501, 504, and 505 during Legs 68, 69 and 70 of the Glomar Challenger are the most depleted in the most-hygromagmaphile elements ( $\mathrm{Th}, \mathrm{Ta}$, $\mathrm{Nb}$, and $\mathrm{La}$ ) of all MORB recovered to date by the Glomar Challenger. The invariant ratios $\mathrm{Nb} / \mathrm{Ta}, \mathrm{Zr} / \mathrm{Hf}$, and $\mathrm{Y} / \mathrm{Tb}$ show "chondritic values" (expected for $\mathrm{Nb} / \mathrm{Ta}$ because of the very low concentrations in these elements). Four samples from a single unit are exceptions: they present a flat to slightly enriched, extended Coryell-Masuda plot, and at the same time their $\mathrm{La} / \mathrm{Ta}$ ratio is 9 (normalized ratio $=1$ ) instead of 19 (normalized ratio $=2$ ), the value for all other samples. Only one of these two values of the $\mathrm{La}$ /Ta ratio had been found so far within a single hole, and moreover within large areas of the oceanic crust (several holes or dredges). The present result shows that local heterogeneity of the upper mantle with respect to the $\mathrm{La} / \mathrm{Ta}$ ratio may exist. ${ }^{5}$
\end{abstract}

\section{INTRODUCTION}

Six holes $(501,504 \mathrm{~A}, 504 \mathrm{~B}, 505,505 \mathrm{~A}, 505 \mathrm{~B})$ were drilled during Legs 68,69 , and 70 of the Glomar Challenger, in a limited area of the Costa Rica Rift: Site 501 $\left(1^{\circ} 13.63^{\prime} \mathrm{N}, 83^{\circ} 44.06^{\prime} \mathrm{W}\right)$; Site $504\left(1^{\circ} 13.6^{\prime} \mathrm{N}, 83^{\circ} 44^{\prime}\right.$ W); Site $505\left(1^{\circ} 54.8^{\prime} \mathrm{N}, 83^{\circ} 47.4^{\prime} \mathrm{W}\right)$. Trace-element data obtained by neutron activation analysis (NAA) and by X-ray fluorescence spectrometry (XRF) are presented in this paper. These data are compared to major-element analyses and sample descriptions obtained on board, and allow us to refine the characterization and classification of the different basaltic units. We, then, interpret them in terms of high- and low-partition-coefficient elements. The behavior of low-partition-coefficient elements (hygromagmaphile elements) is discussed on the basis of comparative geochemistry by using an extended Coryell-Masuda plot, including both rare-earth and other trace elements.

\section{ANALYTICAL CHEMISTRY, RESULTS, AND INVARIANT RATIOS}

The XRF analytical procedure used on board for the measurements of concentrations of major elements is the classical heavy-absorber method. The specific arrangement for shipboard determinations was described by Bougault (1977a), and some modifications were made during leg 45 (Melson et al., 1978). The major-element data are given in Table 1.

The trace-element data (Table 2) are arranged in the order of increasing atomic number. Blank lines separate

\footnotetext{
${ }^{1}$ Cann, J. R,. Langseth, M. G., Honnorez, J., Von Herzen, R. P., White, S. M., et al., Init. Repts. DSDP, 69: Washington (U.S. Govt. Printing Office).

2 CNEXO-COB, BP 337, 29273 Brest, Cedex, France.

3 Institut de Physique du Globe de Paris, T14, E3, 4, place Jussieu, 75230 Paris, Cedex, France.

${ }^{4}$ Laboratoire de Géochimie systématique et comparée, Université Paris VI, 2, place Jussieu, 75230 Paris, Cedex 05, France.

5 Measurements on samples of Hole 504B category 3 (Samples 504B-18-1, 84-88 cm; 504B-18-2, 98-102 cm; 504B-19-1, 73-77 cm; 504B-19-2, 46-49 cm) and category 2 (Samples 504B-54-1, 16-18 cm; 504B-56-1, 139-142 cm; 504B-57-1, 51-55 cm) have been completed since the text was written. The value $16 \pm 1$ of the $\mathrm{Nb} / \mathrm{Ta}$ ratio is confirmed for all of these since the text was written. The value $16 \pm 1$ of the $\mathrm{Nb} / \mathrm{Ta}$ ratio is confirmed for all of these
samples. The normalized $\mathrm{La} / \mathrm{Ta}(\mathrm{La} / \mathrm{Nb})$ ratio of category 2 is about 2 , and this normalized ratio is confirmed for category 3 . (See text.)
}

the homogeneous sets of data versus depth corresponding to the different basalt lithologic units. A brief look at these data shows that four hygromagmatophile elements (Th, Nb, Ta, La, which behave with low solid/liquid bulk partition coefficients) have very low concentrations. For these four elements, the concentrations are close to the limits of detection. It is therefore necessary to discuss accuracy of the appropriate analytical method before interpreting these data with confidence.

In order of increasing atomic number, all the element concentrations up to $\mathrm{Nb}$ have been determined by XRF, except Sc, which was analyzed by NAA, and $\mathrm{Co}$ and $\mathrm{Ni}$, for which both XRF and NAA data are available. From $\mathrm{Sb}$ to $\mathrm{Th}$, all concentrations have been measured by NAA. XRF interferences are corrected, where necessary, according the procedure described by Bougault et al. (1977b). In the case of $\mathrm{Nb}$, there is no instrumental interference; the accuracy of $\mathrm{Nb}$ determination is then limited by the method of calculation of the matrix effects and the measurements of both peak and background intensity. For low $\mathrm{Nb}$ concentrations, close to the 1-ppm detection limit, the accuracy is limited mainly by the counting statistics of both peak and background intensities, and by the method of measuring and substracting the background. NAA measurements are made with a $\mathrm{Ge}-\mathrm{Li}$ detector from 4 days to 1 month after irradiation (Jaffrezic et al., 1977). For low concentration levels, the accuracy also is limited by the counting statistics of intensities and the method of measuring and substracting the background. Thus, for both methods, the accuracy can be considered to be the sum of two terms. The first one, the precision, $d x$, presents a random character reflecting the counting statistics, and can be written $\pm d x$. The second term, $\Delta X$, is constant and can be positive or negative; it corresponds, for instance, to the method of substracting the background. $\Delta X$ corresponds to a systematic error which cannot be identified, despite the care taken in the calibration (blanks, standards, etc.). Any measurement has to be written $X+\Delta X \pm d x$. The value $d x$ can be improved by repeating the measurements. As an example, the points in Figure 1 represent 


\section{J. ETOUBLEAU ET AL.}

Table 1. Major-element analyses.

\begin{tabular}{|c|c|c|c|c|}
\hline Sample & & & & \\
\hline (interval in $\mathrm{cm}$ ) & $\mathrm{SiO}_{2}$ & $\mathrm{TiO}_{2}$ & $\mathrm{Al}_{2} \mathrm{O}_{3}$ & $\mathrm{Fe}_{2} \mathrm{O}_{3}$ \\
\hline $501-10-1,12-16$ & 49.64 & 1.03 & 14.95 & 11.26 \\
\hline $10-1,120-124$ & 49.45 & 1.07 & 15.23 & 10.60 \\
\hline $11-1,85-88$ & 49.88 & 1.04 & 14.73 & 11.91 \\
\hline $11-2,87-89$ & 49.65 & 1.07 & 14.61 & 11.89 \\
\hline $12-1,12-9$ & 49.23 & 1.11 & 15.40 & 11.42 \\
\hline $12-1,59-62$ & 50.27 & 1.14 & 15.85 & 10.44 \\
\hline $13-1,47-49$ & 49.72 & 1.05 & 15.01 & 10.51 \\
\hline $13-2,112-114$ & 49.81 & 1.06 & 14.84 & 10.74 \\
\hline $14-1,10-25$ & 50.05 & 1.04 & 14.62 & 11.28 \\
\hline $14-1,59-62$ & 50.62 & 1.03 & 14.72 & 11.28 \\
\hline $14-2,121-126$ & 50.47 & 1.06 & 14.77 & 11.06 \\
\hline $14-3,135-138$ & 50.00 & 0.87 & 16.07 & 9.67 \\
\hline $15-1,135-138$ & 49.68 & 0.87 & 16.08 & 10.10 \\
\hline $15-4,18-19$ & 49.58 & 0.87 & 15.92 & 9.63 \\
\hline $16-1,118-120$ & 49.05 & 0.85 & 16.30 & 9.80 \\
\hline $16-1,118-120$ & 49.37 & 0.86 & 16.29 & 9.64 \\
\hline $17-1,86-89$ & 50.01 & 0.89 & 15.83 & 9.92 \\
\hline $18-1,119-124$ & 49.15 & 0.89 & 15.81 & 10.27 \\
\hline $18-2,34-37$ & 49.47 & 0.93 & 15.23 & 10.81 \\
\hline $18-2,89-93$ & 50.63 & 1.02 & 14.80 & 11.02 \\
\hline $18-3,100-103$ & 49.97 & 0.91 & 16.04 & 10.11 \\
\hline $19-1,68-71$ & 49.83 & 1.04 & 17.39 & 9.24 \\
\hline $19-2,124-127$ & 49.41 & 0.96 & 15.47 & 10.54 \\
\hline $20-2,137-141$ & 49.89 & 0.90 & 14.93 & 10.88 \\
\hline $20-3,81-84$ & 49.75 & 0.90 & 15.06 & 10.96 \\
\hline $504 A-6-1,64-67$ & 50.29 & 1.05 & 14.60 & 11.28 \\
\hline $6-2,96-98$ & 49.87 & 1.06 & 15.18 & 11.01 \\
\hline $6-3,63-65$ & 50.45 & 1.11 & 15.18 & 10.21 \\
\hline $7-1,75-77$ & 49.56 & 1.09 & 14.98 & 11.20 \\
\hline $7-2,51-53$ & 50.70 & 1.12 & 15.45 & 9.22 \\
\hline $504 \mathrm{~B}-2-1,107-110$ & 50.63 & 1.11 & 15.59 & 9.46 \\
\hline $3-1,102-105$ & 49.63 & 1.07 & 14.98 & 11.70 \\
\hline $4-1,32-38$ & 49.76 & 0.88 & 16.04 & 9.66 \\
\hline $4-2,52-58$ & 49.61 & 0.90 & 16.22 & 9.86 \\
\hline $4-3,27-30$ & 50.13 & 0.88 & 15.84 & 9.77 \\
\hline $4-4,83-87$ & 49.83 & 0.88 & 16.18 & 9.76 \\
\hline $4-5,36-41$ & 49.47 & 0.91 & 16.09 & 9.91 \\
\hline $5-1,86-92$ & 49.15 & 0.89 & 15.88 & 10.07 \\
\hline $5-2,110-115$ & 49.52 & 0.90 & 16.55 & 9.22 \\
\hline $6-1,79-80$ & 49.73 & 0.92 & 16.46 & 9.86 \\
\hline $6-1,88-90$ & 53.28 & 0.11 & 6.38 & 10.83 \\
\hline $6-2,98-105$ & 49.48 & 0.93 & 15.41 & 10.49 \\
\hline $7-1,91-93$ & 50.02 & 0.93 & 16.69 & 9.23 \\
\hline $7-2,91-96$ & 49.25 & 0.88 & 15.86 & 9.89 \\
\hline $7-3,116-120$ & 50.38 & 0.91 & 15.93 & 9.90 \\
\hline $7-4,64-69$ & 49.51 & 0.89 & 16.16 & 10.03 \\
\hline $7-5,71-74$ & 50.47 & 0.91 & 16.22 & 9.40 \\
\hline $8-1,109-115$ & 49.59 & 0.90 & 16.29 & 9.84 \\
\hline $8-2,125-131$ & 50.08 & 0.88 & 16.25 & 9.37 \\
\hline $8-3,113-116$ & 49.43 & 0.88 & 16.54 & 9.99 \\
\hline $8-4,120-124$ & 50.53 & 0.89 & 16.27 & 8.79 \\
\hline $9-1,54-59$ & 49.76 & 0.88 & 15.96 & 9.84 \\
\hline $9-2,63-68$ & 49.25 & 0.98 & 16.29 & 10.12 \\
\hline $10-1,53-58$ & 49.99 & 1.03 & 16.31 & 9.44 \\
\hline $10-2,113-118$ & 50.32 & 0.98 & 15.46 & 9.77 \\
\hline $11-1,16-21$ & 50.59 & 0.98 & 15.51 & 9.50 \\
\hline $11-2,86-95$ & 50.56 & 0.99 & 15.40 & 9.63 \\
\hline $11-3,74-83$ & 50.33 & 0.98 & 15.32 & 9.18 \\
\hline $12-2,123-127$ & 50.25 & 0.96 & 15.07 & 9.79 \\
\hline $13-1,135-140$ & 50.38 & 0.95 & 15.21 & 9.81 \\
\hline $13-2,113-119$ & 50.44 & 0.99 & 15.34 & 9.39 \\
\hline $13-3,87-94$ & 50.07 & 0.96 & 15.09 & 10.15 \\
\hline $13-4,44-49$ & 50.95 & 0.97 & 15.73 & 8.51 \\
\hline $14-2,34-40$ & 50.38 & 0.92 & 15.43 & 9.98 \\
\hline $15-1,125-129$ & 50.00 & 0.91 & 14.64 & 10.71 \\
\hline $15-2,38-44$ & 50.04 & 0.95 & 15.21 & 10.31 \\
\hline $15-3,133-137$ & 50.06 & 0.92 & 15.35 & 9.34 \\
\hline $15-4,58-62$ & 50.10 & 0.92 & 14.49 & 10.80 \\
\hline $15-5,17-22$ & 50.16 & 0.93 & 15.26 & 10.26 \\
\hline $16-1,141-143$ & 49.21 & 0.98 & 14.63 & 11.36 \\
\hline $16-2,89-92$ & 49.32 & 0.82 & 15.48 & 10.93 \\
\hline $16-3,129-139$ & 49.21 & 0.90 & 15.59 & 9.76 \\
\hline $16-5,21-26$ & 49.41 & 0.94 & 15.96 & 9.42 \\
\hline $17-1,3-6$ & 53.52 & 0.03 & 6.48 & 10.63 \\
\hline $17-1,110-114$ & 49.27 & 0.91 & 15.69 & 9.89 \\
\hline $17-2,94-97$ & 49.65 & 0.92 & 15.94 & 9.90 \\
\hline $17-3,22-24$ & 49.93 & 0.95 & 15.70 & 9.86 \\
\hline $18-1,84-98$ & 49.13 & 1.24 & 16.85 & 10.19 \\
\hline $18-2,98-102$ & 49.95 & 1.33 & 16.72 & 8.44 \\
\hline $19-1,73-77$ & 49.26 & 1.37 & 17.22 & 9.43 \\
\hline $19-2,46-49$ & 49.69 & 1.23 & 17.41 & 9.04 \\
\hline $19-2,88-92$ & 49.71 & 0.86 & 16.16 & 9.88 \\
\hline $20-1,142-145$ & 49.63 & 1.01 & 16.21 & 10.07 \\
\hline $21-1,70-75$ & 49.66 & 1.00 & 16.59 & 9.79 \\
\hline $21-2,101-105$ & 49.60 & 0.76 & 16.76 & 9.45 \\
\hline $21-3,110-115$ & 50.05 & 0.99 & 15.85 & 9.66 \\
\hline $21-5,43-46$ & 49.81 & 0.74 & 16.09 & 9.72 \\
\hline $22-1,25-28$ & 49.29 & 0.70 & 16.23 & 9.24 \\
\hline $22-2,56-62$ & 49.62 & 0.74 & 16.60 & 9.26 \\
\hline $23-1,112-117$ & 49.79 & 0.88 & 16.42 & 9.77 \\
\hline $24-1,62-66$ & 49.48 & 0.85 & 15.96 & 9.69 \\
\hline $24-2,85-88$ & 49.62 & 0.88 & 15.82 & 9.77 \\
\hline
\end{tabular}


Table 1. (Continued).

\begin{tabular}{|c|c|c|c|c|c|c|c|c|c|c|c|c|}
\hline \multirow{2}{*}{$\begin{array}{c}\text { Sample } \\
\text { (interval in } \mathrm{cm} \text { ) }\end{array}$} & \multirow[b]{2}{*}{$\mathrm{SiO}_{2}$} & \multirow[b]{2}{*}{$\mathrm{TiO}_{2}$} & \multirow[b]{2}{*}{$\mathrm{Al}_{2} \mathrm{O}_{3}$} & \multirow[b]{2}{*}{$\mathrm{Fe}_{2} \mathrm{O}_{3}$} & & Comp & nent & & & & & \\
\hline & & & & & $\mathrm{MnO}$ & $\mathrm{MgO}$ & $\mathrm{CaO}$ & $\mathrm{Na}_{2} \mathrm{O}$ & $\mathrm{K}_{2} \mathrm{O}$ & $\mathrm{P}_{2} \mathrm{O}_{5}$ & Total & LOI \\
\hline $504 \mathrm{~B}-24-3,104-106$ & 49.57 & 1.00 & 16.19 & 9.52 & 0.18 & 8.54 & 12.89 & 1.99 & 0.15 & 0.08 & 100.11 & 0.83 \\
\hline $25-1,94-98$ & 50.20 & 0.99 & 14.71 & 10.56 & 0.19 & 8.37 & 12.84 & 2.01 & 0.09 & 0.08 & 100.04 & 0.18 \\
\hline $25-2,96-99$ & 50.87 & 1.01 & 15.13 & 9.24 & 0.16 & 8.64 & 12.92 & 2.38 & 0.11 & 0.07 & 100.55 & 0.63 \\
\hline $26-1,52-54$ & 50.36 & 1.00 & 15.06 & 9.98 & 0.18 & 8.43 & 12.71 & 2.25 & 0.17 & 0.07 & 100.21 & 0.54 \\
\hline $27-1,130-134$ & 49.60 & 1.12 & 17.68 & 9.07 & 0.16 & 6.39 & 13.34 & 2.95 & 0.09 & 0.10 & 100.50 & 1.41 \\
\hline $28-1,110-116$ & 50.22 & 0.94 & 15.27 & 10.38 & 0.16 & 8.05 & 12.94 & 1.90 & 0.18 & 0.08 & 100.12 & 0.94 \\
\hline $28-2,39-43$ & 49.51 & 1.09 & 16.92 & 10.06 & 0.17 & 6.75 & 12.93 & 2.29 & 0.10 & 0.11 & 99.93 & 1.65 \\
\hline $28-3,33-37$ & 49.91 & 0.93 & 16.10 & 9.82 & 0.18 & 7.94 & 13.12 & 1.92 & 0.04 & 0.08 & 100.04 & 0.43 \\
\hline $28-3,35-40^{\mathrm{C}}$ & 53.76 & 0.03 & 6.07 & 11.35 & 0.06 & 25.19 & 1.31 & 2.09 & 0.15 & 0.03 & 100.04 & 6.53 \\
\hline $28-4,23-27$ & 49.26 & 0.93 & 15.96 & 10.11 & 0.19 & 7.85 & 13.19 & 1.86 & 0.09 & 0.09 & 99.53 & 0.40 \\
\hline $28-5,6-9$ & 50.08 & 0.91 & 15.99 & 9.49 & 0.17 & 8.07 & 13.21 & 1.94 & 0.02 & 0.08 & 99.96 & 0.97 \\
\hline $29-2,11-16$ & 50.49 & 0.99 & 14.90 & 9.78 & 0.17 & 8.49 & 12.83 & 2.36 & 0.15 & 0.08 & 100.24 & 0.96 \\
\hline $32-1,142-145$ & 49.85 & 0.93 & 15.16 & 10.20 & 0.16 & 8.71 & 13.04 & 1.89 & 0.04 & 0.09 & 100.07 & 0.19 \\
\hline $35-1,99-105$ & 48.26 & 0.80 & 17.12 & 9.35 & 0.14 & 8.92 & 13.37 & 1.76 & 0.08 & 0.09 & 99.89 & 0.86 \\
\hline $36-1,11-16$ & 49.88 & 0.89 & 15.93 & 9.42 & 0.15 & 8.81 & 13.07 & 1.87 & 0.08 & 0.11 & 100.21 & 0.73 \\
\hline $36-2,59-64$ & 49.71 & 0.93 & 14.95 & 10.44 & 0.16 & 8.77 & 12.82 & 1,84 & 0.06 & 0.11 & 99.79 & n.d. \\
\hline $36-3,8-12$ & 49.69 & 0.79 & 16.11 & 9.19 & 0.14 & 9.46 & 13.02 & 1.75 & 0.08 & 0.12 & 100.35 & 0.44 \\
\hline $36-3,123-127$ & 50.04 & 0.94 & 15.05 & 10.33 & 0.16 & 8.45 & 12.80 & 1.86 & 0.06 & 0.11 & 99.80 & 0.52 \\
\hline $36-4,109-114$ & 50.05 & 0.91 & 14.87 & 10.38 & 0.16 & 8.27 & 12.83 & 1.91 & 0.08 & 0.10 & 99.56 & 1.10 \\
\hline $39-1,77-80$ & 49.86 & 0.96 & 15.95 & 9.66 & 0.16 & 8.45 & 12.50 & 2.12 & 0.11 & 0.10 & 99.87 & 0.71 \\
\hline $39-2,24-28$ & 50.10 & 0.92 & 15.24 & 10.31 & 0.16 & 8.31 & 13.10 & 1.89 & 0.05 & 0.11 & 100.19 & 0.08 \\
\hline $39-3,53-55$ & 50.00 & 0.81 & 16.29 & 9.60 & 0.14 & 8.57 & 12.90 & 1.60 & 0.08 & 0.09 & 100.08 & 0.35 \\
\hline $40-1,14-17$ & 50.02 & 0.80 & 16.27 & 9.30 & 0.14 & 8.40 & 12.96 & 1.76 & 0.07 & 0.11 & 99.83 & 1.25 \\
\hline $40-1,138-141$ & 49.71 & 0.80 & 16.25 & 9.25 & 0.14 & 8.76 & 12.99 & 1.96 & 0.07 & 0.10 & 100.03 & 0.51 \\
\hline $40-3,80-83$ & 49.97 & 0.80 & 16.16 & 9.25 & 0.15 & 8.93 & 13.14 & 1.62 & 0.04 & 0.11 & 100.17 & 1.06 \\
\hline $40-3,139-141$ & 50.67 & 0.74 & 16.66 & 8.95 & 0.14 & 7.86 & 13.62 & 1.80 & 0.07 & 0.10 & 100.61 & 0.50 \\
\hline $40-4,67-72$ & 50.25 & 0.79 & 16.66 & 9.17 & 0.14 & 8.68 & 13.17 & 1.91 & 0.06 & 0.12 & 100.95 & 0.21 \\
\hline $40-5,31-34$ & 50.47 & 0.80 & 15.98 & 9.37 & 0.15 & 8.48 & 12.94 & 1.72 & 0.08 & 0.10 & 100.09 & 0.83 \\
\hline $41-1,128-130$ & 49.64 & 0.81 & 16.30 & 9.34 & 0.15 & 8.67 & 13.03 & 1.76 & 0.07 & 0.10 & 99.87 & 1.04 \\
\hline $41-2,25-30$ & 49.71 & 0.80 & 16.26 & 9.32 & 0.14 & 8.94 & 12.90 & 1.68 & 0.05 & 0.10 & 99.90 & 1.27 \\
\hline $41-2,136-138$ & 50.08 & 0.83 & 15.91 & 9.60 & 0.15 & 9.02 & 12.95 & 1.77 & 0.12 & 0.10 & 100.53 & 0.74 \\
\hline $42-2,40-44$ & 50.13 & 0.86 & 15.86 & 9.43 & 0.15 & 8.71 & 13.02 & 1.99 & 0.11 & 0.10 & 100.36 & 1.43 \\
\hline $44-1,25-28$ & 49.63 & 1.38 & 14.96 & 10.34 & 0.16 & 8.24 & 12.80 & 2.23 & 0.12 & 0.18 & 100.04 & 1.20 \\
\hline $44-2,93-96$ & 50.04 & 0.91 & 15.70 & 10.37 & 0.16 & 7.98 & 13.30 & 1.68 & 0.07 & 0.10 & 100.31 & 1.10 \\
\hline $45-1,85-88$ & 50.18 & 0.90 & 16.01 & 9.82 & 0.16 & 8.02 & 13.29 & 2.10 & 0.10 & 0.10 & 100.68 & 1.04 \\
\hline $45-2,91-96$ & 50.24 & 0.96 & 15.81 & 10.33 & 0.16 & 8.41 & 12.61 & 1.90 & 0.07 & 0.11 & 100.60 & 0.60 \\
\hline $47-1,43-45$ & 50.44 & 0.99 & 14.30 & 11.04 & 0.17 & 8.57 & 12.29 & 1.92 & 0.13 & 0.11 & 99.96 & 0.08 \\
\hline $47-2,20-23$ & 50.63 & 1.00 & 14.09 & 11.52 & 0.18 & 8.40 & 12.20 & 1.87 & 0.12 & 0.13 & 100.14 & 1.12 \\
\hline $47-2,103-108$ & 50.16 & 0.96 & 14.93 & 10.74 & 0.15 & 8.40 & 12.65 & 1.73 & 0.14 & 0.11 & 99.97 & 1.12 \\
\hline $47-3,24-27$ & S0.18 & 0.99 & 14.90 & 10.80 & 0.16 & 8.59 & 12.68 & 2.04 & 0.10 & 0.10 & 100.54 & 0.90 \\
\hline $48-2,80-84$ & 50.68 & 0.99 & 14.54 & 10.94 & 0.17 & 8.44 & 12.47 & 1.95 & 0.12 & 0.11 & 100.41 & 0.81 \\
\hline $48-3,97-100$ & 49.64 & 0.87 & 16.14 & 9.64 & 0.15 & 8.55 & 13.24 & 1.71 & 0.04 & 0.10 & 100.08 & 0.64 \\
\hline $50-1,68-72$ & 49.80 & 0.84 & 16.08 & 9.50 & 0.15 & 8.60 & 13.20 & 2.20 & 0.09 & 0.10 & 100.56 & 1.70 \\
\hline $51-1,132-135$ & 50.35 & 0.85 & 15.07 & 9.37 & 0.15 & 8.72 & 13.20 & 1.86 & 0.07 & 0.12 & 99.76 & 1.80 \\
\hline $52-1,62-65$ & 50.13 & 0.85 & 15.59 & 9.41 & 0.15 & 8.47 & 13.55 & 1.88 & 0.08 & 0.11 & 100.22 & 1.12 \\
\hline $52-2,55-60$ & 50.22 & 0.85 & 15.99 & 9.48 & 0.15 & 8.49 & 13.14 & 1.69 & 0.12 & 0.10 & 100.23 & 0.20 \\
\hline $52-3,100-103$ & 50.17 & 0.85 & 15.90 & 9.38 & 0.15 & 8.77 & 13.12 & 1.82 & 0.08 & 0.09 & 100.33 & 1.35 \\
\hline $52-4,63-66$ & 50.03 & 0.87 & 15.68 & 9.63 & 0.15 & 8.84 & 13.03 & 1.67 & 0.10 & 0.10 & 100.10 & 1.50 \\
\hline $54-1,16-18$ & 49.94 & 0.93 & 15.58 & 9.90 & 0.16 & 8.35 & 13.22 & 1.98 & 0.07 & 0.10 & 100.23 & 0.96 \\
\hline $56-1,139-142$ & 49.65 & 1.37 & 14.65 & 10.59 & 0.17 & 8.59 & 12.41 & 2.13 & 0.05 & 0.18 & 99.79 & 0.57 \\
\hline $57-1,51-55$ & 50.06 & 1.36 & 14.90 & 10.12 & 0.16 & 8.54 & 12.37 & 2.25 & 0.11 & 0.18 & 100.05 & 1.25 \\
\hline $57-2,26-30$ & 49.90 & 0.98 & 15.31 & 9.90 & 0.16 & 8.86 & 12.70 & 2.10 & 0.04 & 0.14 & 100.09 & 0.36 \\
\hline $58-1,141-146$ & 50.15 & 0.91 & 15.92 & 9.85 & 0.16 & 8.18 & 13.04 & 1.86 & 0.09 & 0.12 & 100.28 & 1.22 \\
\hline $58-3,14-17$ & 50.04 & 0.92 & 15.61 & 10.02 & 0.16 & 8.31 & 13.08 & 1.88 & 0.08 & 0.11 & 100.21 & 0.95 \\
\hline $59-1,46-48$ & 49.87 & 1.02 & 15.58 & 9.98 & 0.16 & 8.49 & 12.78 & 1.90 & 0.14 & 0.12 & 100.04 & 1.17 \\
\hline $60-1,50-54$ & 50.28 & 1.06 & 14.86 & 10.80 & 0.17 & 8.73 & 12.70 & 1.85 & 0.06 & 0.12 & 100.63 & 1.22 \\
\hline $61-1,112-115$ & 49.39 & 1.05 & 15.20 & 11.20 & 0.18 & 8.28 & 13.17 & 1.72 & 0.07 & 0.14 & 100.40 & 1.37 \\
\hline $63-2,103-105$ & 50.23 & 1.03 & 14.65 & 10.80 & 0.17 & 8.73 & 12.71 & 1.70 & 0.07 & 0.10 & 100.19 & 1.03 \\
\hline $64-2,107-109$ & 50.35 & 1.18 & 13.83 & 11.82 & 0.18 & 8.44 & 11.75 & 2.03 & 0.04 & 0.11 & 99.73 & 1.28 \\
\hline $64-4,78-82$ & 50.07 & 1.00 & 15.27 & 11.02 & 0.17 & 8.49 & 12.60 & 1.80 & 0.08 & 0.12 & 100.62 & 1.21 \\
\hline $70-1,56-60$ & 50.31 & 1.10 & 14.25 & 11.53 & 0.19 & 8.79 & 11.96 & 1.79 & 0.05 & 0.11 & 100.08 & 1.06 \\
\hline $505-25-1,26-28$ & 48.45 & 0.71 & 17.44 & 8.15 & 0.13 & 9.67 & 13.22 & 1.64 & 0.08 & 0.08 & 99.57 & 0.78 \\
\hline $26-1,33-36$ & 48.03 & 0.76 & 17.46 & 8.25 & 0.14 & 9.07 & 13.43 & 1.88 & 0.09 & 0.10 & 99.21 & 0.94 \\
\hline $505 \mathrm{~A}-1-1,28-30$ & 49.13 & 0.95 & 16.16 & 9.74 & 0.16 & 8.53 & 12.79 & 2.00 & 0.07 & 0.09 & 99.62 & 0.23 \\
\hline $2-1,51-55$ & 48.93 & 0.95 & 15.94 & 9.69 & 0.15 & 8.82 & 12.60 & 2.32 & 0.04 & 0.08 & 99.52 & 0.95 \\
\hline 505B-2-1, 19-23 & 49.58 & 0.98 & 15.94 & 9.59 & 0.15 & 8.92 & 12.69 & 2.08 & 0.06 & 0.08 & 100.07 & 1.04 \\
\hline $2-2,87-90$ & 49.63 & 0.96 & 16.23 & 9.34 & 0.14 & 8.40 & 12.89 & 1.98 & 0.08 & 0.08 & 99.73 & 1.20 \\
\hline $2-3,51-52$ & 49.70 & 0.96 & 16.24 & 9.64 & 0.18 & 7.72 & 12.89 & 2.46 & 0.17 & 0.09 & 100.05 & 1.71 \\
\hline $2-3,78-83$ & 49.62 & 0.96 & 16.05 & 9.36 & 0.14 & 8.89 & 12.63 & 2.17 & 0.05 & 0.08 & 99.95 & 1.17 \\
\hline $3-1,76-79$ & 49.52 & 0.95 & 16.21 & 9.54 & 0.15 & 8.71 & 12.56 & 2.19 & 0.13 & 0.08 & 100.04 & 0.33 \\
\hline $3-2,102-103$ & 49.67 & 0.95 & 15.94 & 9.51 & 0.14 & 8.70 & 12.57 & 2.11 & 0.06 & 0.08 & 99.73 & 0.96 \\
\hline $5-1,40-43$ & 49.63 & 0.97 & 16.60 & 9.37 & 0.14 & 8.53 & 12.64 & 2.12 & 0.07 & 0.08 & 100.15 & 0.96 \\
\hline $6-1,114-116$ & 50.04 & 0.97 & 15.90 & 9.44 & 0.15 & 8.34 & 12.85 & 2.17 & 0.05 & 0.09 & 100.00 & 1.01 \\
\hline $6-2,20-23$ & 49.64 & 0.97 & 15.79 & 9.72 & 0.15 & 9.15 & 12.61 & 2.11 & 0.06 & 0.08 & 100.28 & 0.57 \\
\hline
\end{tabular}

the average values $(\mathrm{Nb}, \mathrm{Ta})$ for the various basaltic units encountered in the Gulf of California at $22^{\circ} \mathrm{N}$, Sites 482,483 , and 485 , drilled during Leg 65 ; the indicated error bars correspond to $d x$. The $d x$ value of a single determination is about $1.5 \mathrm{ppm}$ for $\mathrm{Nb}$. The $d x$ values indicated in Figure 1 are lower, and account for several determinations of different samples of the same basaltic unit. By definition, there is no way to improve the $\Delta X$ value. When $X$ is low, and of the order of magnitude of the estimated value of $\Delta X$, it is not possible to compute a ratio of concentrations $(\mathrm{Nb} / \mathrm{Ta}$, for instance) from a single pair of values. Nevertheless, it is still possible to estimate such a ratio from a least-squares calculation, provided that the number of points is sufficient, and that they lie in a range of values larger, at least by a factor two, than the $d x$ precision values which account for the reproducibility of measurements. The $\mathrm{Nb}$ and $\mathrm{Ta}$ data for Leg 65 (Fig. 1) fulfill these requirements. The 
Table 2. Trace-element analyses.

\begin{tabular}{|c|c|c|c|c|c|c|c|c|c|c|c|c|c|c|}
\hline & & & & & & & & & & & & Elem & & \\
\hline in $\mathrm{cm}$ ) & $\mathrm{Sc}$ & $\mathrm{Ti}$ & $\mathrm{v}$ & $\mathrm{Cr}$ & Mn & $\mathrm{Fe}$ & $\mathrm{Co}_{\mathrm{x}}$ & $\mathrm{Co}_{\mathrm{n}}$ & $\mathrm{Ni}_{\mathrm{x}}$ & $\mathrm{Ni}_{\mathrm{n}}$ & $\mathrm{Zn}$ & $\mathrm{Rb}$ & Sr & $\mathbf{Y}$ \\
\hline $501-10-1,12-16$ & & 6180 & 357 & 234 & 1471 & 78820 & 44 & n.d. & 78 & n.d. & 76 & 1.4 & 65 & 32 \\
\hline $10-1,120-124$ & .8 & 20 & 365 & 15 & 1394 & & 47 & & n.d. & & 82 & & 67 & \\
\hline $11-1,85-88$ & 43.6 & 6240 & 357 & 220 & 1471 & 83370 & 46 & 45.6 & n.d. & 71 & 77 & & 64 & 34 \\
\hline $11-2,87-89$ & n.d. & 6420 & 362 & 207 & 1394 & 83230 & 43 & n.d. & 79 & n.d. & n.d. & 6 & 66 & \\
\hline & & & 0 & 12 & 94 & 0 & 40 & n.d. & 60 & n.d. & 74 & 8 & 70 & \\
\hline & & 6840 & 384 & 230 & 1162 & 73080 & 42 & n.d. & 77 & n.d. & 82 & 4 & 76 & 32 \\
\hline $13-1,47-49$ & n.d. & 6300 & 357 & 202 & 1162 & 73570 & 45 & n.d. & 120 & n.d. & 72 & 0.5 & 65 & 31 \\
\hline & & & 364 & 214 & & 75180 & 46 & n.d. & 113 & & 70 & 0.0 & 65 & 32 \\
\hline 14. & & & 335 & 91 & 1316 & 78960 & 43 & n.d. & 110 & d. & 67 & .0 & 64 & 32 \\
\hline & & & 341 & 187 & 1316 & 78960 & 44 & n.d. & 106 & n.d. & 71 & 1.4 & 65 & 32 \\
\hline $14-2,121-126$ & 44.3 & 6360 & 360 & 203 & 1316 & 77420 & 47 & 47.3 & 107 & 87 & 73 & 1.5 & 65 & 30 \\
\hline $14-3,135-138$ & 38.1 & 5220 & 291 & 446 & 1471 & 67690 & 43 & 43.4 & n.d. & 137 & 65 & 1.5 & 66 & 29 \\
\hline $15-1,135-138$ & & 5220 & 280 & 455 & 1394 & 70700 & 42 & 42.1 & 143 & 129 & 60 & 2.8 & 66 & 28 \\
\hline $15-4,18-19$ & .8 & 5220 & 281 & 457 & 1316 & 67410 & 43 & & 31 & 13 & 64 & 0.9 & 62 & 27 \\
\hline & d. & 5100 & 277 & 523 & 16 & 68600 & 42 & & & & 67 & 1.8 & 61 & 6 \\
\hline & & 5160 & 272 & 479 & & 7480 & 41 & & & & & & & \\
\hline $17-1,86-89$ & 39.1 & 5340 & 287 & 421 & 1239 & 69440 & 39 & 41.3 & 140 & & 58 & 0.9 & 61 & 28 \\
\hline $18-1,119-124$ & 40.7 & 5340 & 324 & 283 & 1394 & 71890 & 46 & 48.3 & 175 & 138 & 77 & 1.1 & 53 & 29 \\
\hline & & & 3. & & & 756 & 45 & & & & & & 51 & \\
\hline & & & 61 & & & 771 & 45 & & & & 8 & & 53 & \\
\hline 18-3, & & & 37 & & & 70770 & 45 & & 82 & & 74 & & 54 & \\
\hline $19-1,6$ & & & 3 & & & 64680 & 45 & n.d. & 89 & & 73 & 2.6 & 70 & 31 \\
\hline 19 & & & 53 & & & 73780 & 46 & 47.7 & 100 & & 79 & 3.5 & 45 & 34 \\
\hline $\begin{array}{l}20-2, \\
20-3,\end{array}$ & & $\begin{array}{l}5400 \\
5400\end{array}$ & $\begin{array}{l}333 \\
326\end{array}$ & $\begin{array}{l}286 \\
287\end{array}$ & 1549 & $\begin{array}{l}76160 \\
76720\end{array}$ & $\begin{array}{l}46 \\
51\end{array}$ & $\begin{array}{l}47.9 \\
\text { n.d. }\end{array}$ & 111 & 106 & 72 & 2.0 & 39 & \\
\hline $20-3$ & 43.3 & 6300 & $\begin{array}{l}326 \\
361\end{array}$ & $\begin{array}{l}287 \\
221\end{array}$ & 1471 & $\begin{array}{l}76720 \\
78960\end{array}$ & 46 & $\begin{array}{l}\text { n.d. } \\
46.2\end{array}$ & 150 & n.d. & & & $\begin{array}{l}39 \\
69\end{array}$ & \\
\hline 8 & & 6330 & 382 & 246 & 1394 & 77070 & 49 & & $\begin{array}{l}\begin{array}{l}46 \\
112\end{array} \\
112\end{array}$ & $\begin{array}{r}82 \\
106\end{array}$ & 81 & 4 & 73 & $\begin{array}{l}32 \\
33\end{array}$ \\
\hline & 5.9 & 6660 & 392 & 233 & 1416 & 71470 & 47 & 46.5 & 72 & 73 & 86 & 0.1 & 75 & 35 \\
\hline & & 6540 & 389 & 223 & 1394 & 78400 & 49 & 45.2 & 93 & 91 & 82 & 1. & 76 & 35 \\
\hline $7-2,51-53$ & 47.3 & 6720 & 405 & 241 & 1394 & 64540 & 52 & 55.2 & 122 & 129 & 84 & 0.7 & 78 & 35 \\
\hline $\begin{array}{r}504 \mathrm{~B}-2-1,107-110 \\
3-1,102-105\end{array}$ & $\begin{array}{l}\text { n.d. } \\
\text { n.d. }\end{array}$ & $\begin{array}{l}6660 \\
6420\end{array}$ & $\begin{array}{l}393 \\
383\end{array}$ & $\begin{array}{l}225 \\
215\end{array}$ & & $\begin{array}{l}66220 \\
81900\end{array}$ & $\begin{array}{l}49 \\
45\end{array}$ & & $\begin{array}{r}129 \\
76\end{array}$ & $\begin{array}{l}\text { n.d. } \\
\text { n.d. }\end{array}$ & $\begin{array}{l}86 \\
79\end{array}$ & $\begin{array}{l}1.3 \\
6.2\end{array}$ & $\begin{array}{l}77 \\
73\end{array}$ & $\begin{array}{l}36 \\
32\end{array}$ \\
\hline $\begin{array}{l}4-1,32-38 \\
4-2,52-58\end{array}$ & n.d. & 5280 & 290 & 460 & 1316 & 67620 & 41 & n.d. & 134 & n.d. & 62 & 3.3 & 69 & 27 \\
\hline & & & & & & & 42 & & & & & & 70 & \\
\hline 4 & & & ${ }_{2}^{2}$ & & & & $\begin{array}{l}41 \\
43\end{array}$ & & & & & & 66 & 27 \\
\hline 4 & & & & & & & $\begin{array}{l}43 \\
42\end{array}$ & & & & & & 7 & 2 \\
\hline & n.d. & 5340 & 279 & 445 & & 490 & 40 & & & n & & & 7 & \\
\hline $2,110-115$ & & & 1 & & & & 42 & & & $\mathrm{n}$ & & & 7 & 2 \\
\hline 6 & & 5520 & 311 & 513 & & 69020 & 45 & & 145 & 146 & 6 & & 79 & 19 \\
\hline & 5.7 & 660 & 57 & 31 & 6 & 75810 & 15 & 10.7 & 72 & 85 & 71 & & 74 & 2 \\
\hline 05 & n.d. & 558 & 306 & 444 & 123 & 734 & 39 & & 115 & n.d. & & 4.2 & 69 & 25 \\
\hline 7 & & 55 & 302 & 491 & 13 & 646 & 43 & & 140 & n.d. & 6 & 1. & 74 & 2 \\
\hline 96 & d. & 528 & 273 & 445 & 13 & 692 & 43 & & is & n.d. & & & 66 & 2 \\
\hline-120 & 37.6 & 54 & 283 & 441 & & & 42 & & & & & & 6 & 2 \\
\hline & d. & 53. & 2 & 438 & & & 41 & & & & & & 6 & 2 \\
\hline 7 & & & 2 & & & & 42 & & & & & & & 2 \\
\hline 115 & & & 2 & & & & 37 & & & & & & & \\
\hline 8. & & & 2 & & & & 39 & & 126 & & & & 71 & 2 \\
\hline & & & 2 & & & & 3 & & 99 & & & & 71 & 28 \\
\hline $8-4,120-1$ & & 5340 & 28 & 429 & & & 41 & & 156 & & & & & 2 \\
\hline $9-1,54-58$ & 38.2 & 5280 & 279 & 459 & 1239 & 68880 & 41 & 44.8 & 143 & 159 & 67 & 0.3 & 68 & 27 \\
\hline 2. $63-68$ & 43.5 & 5880 & 345 & 309 & 1239 & 70840 & 49 & 49.8 & 105 & 109 & 79 & 4.1 & 63 & 31 \\
\hline & & & & & & & 4 & & & & & & & \\
\hline & & & 36 & & & & 4 & & 10 & $\mathrm{n}$ & & & & 2 \\
\hline & & & & & & & 4 & & & & & & & \\
\hline & & & 3 & & & & 4 & & & & & & & 2 \\
\hline & & & 3 & & & & 4 & & 108 & & & & 8 & \\
\hline & & & $3 c$ & & & & 44 & & 9 & is & & & $?$ & 25 \\
\hline & & & & & & & & & & & & & & \\
\hline & & & & & & & & & & & & & & \\
\hline & & & & & & & & & & & & & & \\
\hline 1 & & & & & & & & & & & & & & \\
\hline & & & & & & & & & & & & & & \\
\hline & & & & & & & & & & & & & & \\
\hline & & & & & & & 4 & & & n. & & & & \\
\hline & & 55 & 3 & 33 & & & 4 & & & & & & & \\
\hline & & 5520 & 330 & & & & 45 & & & & & & & \\
\hline $15-5,17-22$ & n.d. & 5580 & 327 & 341 & 1394 & 7182 & 44 & n.c & 103 & n.d. & 7. & & & 3 \\
\hline $\begin{array}{l}16-1,141-143 \\
16-2,89-92^{\mathrm{a}}\end{array}$ & n.d. & 5880 & 331 & 322 & 1471 & 7952 & 41 & n.d & 78 & n.d. & 68 & 7.1 & 61 & 31 \\
\hline & & & 28 & & & & & & & & & & & \\
\hline $16-3,129-139$ & 36.5 & 5400 & 255 & 468 & 1394 & 68320 & 42 & 44.6 & 174 & 185 & & 2.0 & & \\
\hline & & & & & & & & & & & & & & \\
\hline & & & & & & & & & & & & & & \\
\hline & & & 261 & 480 & & & & & & & & & & \\
\hline $17-3,22-24$ & 38.2 & 5700 & 287 & 501 & 1471 & 69020 & 48 & 49.9 & 138 & 148 & 69 & 4.1 & 83 & 26 \\
\hline & & & 254 & & & & & & & & & & & \\
\hline & & & & & & & & & & & & & & \\
\hline & & 8220 & 279 & & 1549 & 660 & 39 & & & & & & & \\
\hline $19-2,46-49$ & 36.9 & 7380 & 283 & 408 & 1394 & 63280 & 42 & 43.9 & 123 & 127 & 68 & 1.6 & 159 & 30 \\
\hline $19-2,88-92$ & 37.8 & 5160 & 287 & 443 & 1394 & 69160 & 41 & 40.1 & 121 & 126 & 64 & 4.6 & 68 & 26 \\
\hline $\begin{array}{l}20-1,142-145 \\
21-1,70-75\end{array}$ & $\begin{array}{l}\text { n.d. } \\
\text { n.d. }\end{array}$ & $\begin{array}{l}6060 \\
6000\end{array}$ & $\begin{array}{l}278 \\
286\end{array}$ & $\begin{array}{l}415 \\
415\end{array}$ & $\begin{array}{l}1394 \\
1239\end{array}$ & $\begin{array}{l}70490 \\
68530\end{array}$ & $\begin{array}{l}41 \\
43\end{array}$ & $\begin{array}{l}\text { n.d. } \\
\text { n.d. }\end{array}$ & $\begin{array}{l}114 \\
133\end{array}$ & $\begin{array}{l}\text { n.d. } \\
\text { n.d. }\end{array}$ & $\begin{array}{l}62 \\
66\end{array}$ & $\begin{array}{l}2.5 \\
3.7\end{array}$ & $\begin{array}{l}96 \\
97\end{array}$ & $\begin{array}{l}28 \\
26\end{array}$ \\
\hline & & & & & & & & & & & & & & \\
\hline $21-3,110-115$ & & 5940 & 270 & 419 & 1549 & 67620 & 43 & n.d. & 121 & n.d. & 62 & 0.0 & 93 & 29 \\
\hline $21-5,43-46$ & 37.4 & 4440 & 284 & 407 & 1394 & 68040 & 45 & 45.9 & 139 & 142 & 61 & 2.6 & 55 & 22 \\
\hline
\end{tabular}


Table 2. (Continued).

\begin{tabular}{|c|c|c|c|c|c|c|c|c|c|c|c|c|c|c|c|c|c|c|c|c|c|c|}
\hline & & & & & & & & & & & & Elemet & & & & & & & & & & \\
\hline $\mathrm{val}$ in $\mathrm{cm}$ ) & $\mathrm{Sc}$ & $\mathrm{Ti}$ & v & $\mathrm{Cr}$ & Mn & $\mathrm{Fe}$ & $\mathrm{Co}_{\mathrm{x}}$ & $\mathrm{Co}_{\mathrm{n}}$ & $\mathrm{Ni}_{\mathrm{X}}$ & $\mathrm{Ni}_{\mathrm{n}}$ & $\mathrm{Zn}$ & $\mathbf{R b}$ & $\mathrm{Sr}$ & $\mathbf{Y}$ & $\mathrm{Zr}$ & $\mathrm{Nb}$ & $\mathrm{Sr}$ & Cs & $\mathrm{La}$ & $\mathrm{Eu}$ & $\mathrm{Tb}$ & Hf \\
\hline $504 \mathrm{~B}-22-1,25-28$ & 34.5 & 4200 & 253 & 408 & 1471 & 64680 & 40 & 44.0 & 139 & 146 & 52 & 2.6 & 50 & 22 & 33 & 0.0 & 0.04 & 0.03 & 0.64 & 0.63 & 0.39 & 0.96 \\
\hline $\begin{array}{l}22-2,56-62 \\
23-1,112-117\end{array}$ & $\begin{array}{l}\text { n.d. } \\
39.1\end{array}$ & $\begin{array}{r}4440 \\
5280\end{array}$ & $\begin{array}{l}273 \\
310\end{array}$ & $\begin{array}{l}432 \\
321\end{array}$ & $\begin{array}{l}1316 \\
1316\end{array}$ & $\begin{array}{l}64820 \\
68390\end{array}$ & $\begin{array}{l}40 \\
40\end{array}$ & $\begin{array}{l}\text { n.d. } \\
42.9\end{array}$ & $\begin{array}{l}131 \\
117\end{array}$ & $\begin{array}{l}\text { n.d. } \\
118\end{array}$ & $\begin{array}{l}55 \\
67\end{array}$ & $\begin{array}{l}1.6 \\
2.2\end{array}$ & $\begin{array}{l}53 \\
67\end{array}$ & $\begin{array}{l}23 \\
26\end{array}$ & $\begin{array}{l}36 \\
49\end{array}$ & $\begin{array}{l}0.0 \\
0.8\end{array}$ & $\begin{array}{l}\text { n.d. } \\
0.05\end{array}$ & $\begin{array}{l}\text { n.d. } \\
0.06\end{array}$ & $\begin{array}{l}\text { n.d. } \\
0.87\end{array}$ & $\begin{array}{l}\text { n.d. } \\
0.70\end{array}$ & $\begin{array}{l}\text { n.d. } \\
0.46\end{array}$ & $\begin{array}{l}\text { n.d. } \\
1.25\end{array}$ \\
\hline $24-1,62-66$ & n.d. & 5100 & 274 & 433 & 1394 & 67830 & 42 & n.d. & 132 & n.d. & $\begin{array}{l}07 \\
56\end{array}$ & $\begin{array}{l}2.2 \\
2.2\end{array}$ & $\begin{array}{l}01 \\
63\end{array}$ & $\begin{array}{l}20 \\
25\end{array}$ & $\begin{array}{l}49 \\
46\end{array}$ & $\begin{array}{l}0.8 \\
0.3\end{array}$ & n.d. & n.d. & n.d. & n.d. & n.d. & n.d. \\
\hline $24-2,85-88$ & n.d. & 5280 & 283 & 430 & 1394 & 68390 & 41 & n.d. & 115 & n.d. & 60 & 2.7 & 61 & 25 & 48 & n.d. & n.d. & n.d. & n.d. & n.d. & n.d. & n.d. \\
\hline $24-3,104-106$ & n.d. & 6000 & 291 & 448 & 1396 & 66640 & 42 & n.d. & 118 & n.d. & 71 & 2.9 & 90 & 27 & 60 & 2.0 & n.d. & n.d. & n.d. & n.d. & n.d. & n.d. \\
\hline $25-1,94-98$ & n.d. & 5940 & 323 & 344 & 1471 & 73920 & 44 & n.d. & 93 & n.d. & 72 & 1.9 & 69 & 30 & 54 & 0.6 & n.d. & n.d. & n.d. & n.d. & n.d. & n.d. \\
\hline $25-2,96-99$ & 43.6 & 6060 & 329 & 330 & 1239 & 64680 & 45 & 46.0 & 115 & 115 & 73 & 1.7 & 70 & 31 & 53 & 0.9 & 0.01 & 0.03 & 0.89 & 0.83 & 0.55 & 1.44 \\
\hline $26-1,52-54$ & n.d. & 6000 & 325 & 328 & 1394 & 69860 & 45 & n.d. & 103 & n.d. & 69 & 4.4 & 69 & 32 & 55 & 0.8 & n.d. & n.d. & n.d. & n.d. & n.d. & n.d. \\
\hline $27-1,130-134$ & n.d. & 6720 & 275 & 459 & 1239 & 63490 & 46 & n.d. & 168 & n.d. & 68 & 0.4 & 114 & 30 & 69 & 0.7 & n.d. & n.d. & n.d. & n.d. & n.d. & n.d. \\
\hline $28-1,110-116$ & 42.8 & 5640 & 337 & 275 & 1239 & 72660 & 46 & 47.9 & 114 & 118 & 71 & 3.6 & 52 & 31 & 44 & 0.7 & 0.02 & 0.05 & 0.98 & 0.80 & 0.52 & 1.25 \\
\hline $28-2,39-43$ & n.d. & 6540 & 257 & 443 & 1316 & 70210 & 45 & n.d. & 160 & n.d. & 68 & 2.4 & 108 & 29 & 71 & 0.5 & n.d. & n.d. & n.d. & n.d. & n.d. & n.d. \\
\hline $28-3,33-37$ & n.d. & 5580 & 295 & 330 & 1394 & 68740 & 41 & n.d. & 100 & n.d. & n.d. & 1.9 & 60 & 27 & 45 & 0.0 & n.d. & n.d. & n.d. & n.d. & n.d. & \\
\hline $28-4,23-27$ & n.d. & 5580 & 302 & 302 & 1471 & 70710 & 40 & n.d. & 93 & n.d. & 65 & 2.4 & 60 & 29 & 52 & & & & n.d. & n.d. & n.d. & n.d. \\
\hline $28-5,6-9$ & 38.7 & 5460 & 301 & 334 & 1316 & 66430 & 40 & 42.0 & 107 & 113 & 69 & 0.0 & 59 & 28 & 49 & 1.2 & n.d. & n.d. & 0.91 & 0.73 & 0.48 & 1.40 \\
\hline $29-2,11-16$ & n.d. & 5940 & 301 & 303 & 1316 & 68460 & 43 & n.d. & 103 & n.d. & 67 & 1.9 & 70 & 31 & 52 & & n.d. & n.d. & n.d. & n.d. & & \\
\hline $32-1,141-145$ & n.d. & 5560 & 313 & 328 & 1012 & 71260 & 42 & n.d. & 95 & n.d. & 52 & 1.0 & 60 & 28 & 53 & 1.1 & n.d. & n.d. & n.d. & n.d. & n.d. & n.d. \\
\hline $35-1,99-105$ & n.d. & 4600 & 234 & 413 & 885 & 64890 & 44 & n.d. & 185 & n.d. & 48 & 1.7 & 79 & 24 & 53 & 2.2 & n.d. & n.d. & n.d. & n.d. & n.d. & n.d. \\
\hline $36-1,11-16$ & n.d. & 5280 & 293 & 385 & 948 & 65450 & 37 & n.d. & 102 & n.d. & 47 & 0.7 & 54 & 28 & 45 & 0.0 & n.d. & n.d. & n.d. & n.d. & n.d. & n.d. \\
\hline $36-2,59-66$ & $d$. & 5580 & 301 & 301 & 1012 & 73080 & 41 & n.d. & 94 & n.d. & 51 & 0.7 & 60 & 27 & 53 & 1.1 & n.d. & n.d. & n.d. & n.d. & & n.d. \\
\hline $36-3,8-12$ & 43.3 & 4740 & 330 & 257 & 885 & 64050 & 39 & n.d. & 89 & n.d. & 53 & 0.2 & 61 & 27 & 53 & 1.5 & n.d. & n.d. & 1.00 & 0.80 & 0.54 & 1.35 \\
\hline $36-3,123-127$ & 43.2 & 5640 & 280 & 292 & 1012 & 71960 & 37 & n.d. & 95 & n.d. & 52 & 1.6 & 63 & 29 & so & 1. & n.d. & n.d. & 0.96 & 0.81 & 0.55 & 1.40 \\
\hline $36-4,109-112$ & n.d. & 5400 & 310 & 369 & 1012 & 71890 & 40 & n.d. & 85 & n.d. & 57 & 0.5 & 52 & 28 & 53 & 1.6 & n.d. & n.d. & n.d. & n.d. & n. & n.d. \\
\hline $39-1,77-80$ & n.d. & 5700 & 345 & 466 & 1012 & 67130 & 44 & n.d. & 98 & n.d. & 65 & 0.7 & 64 & 30 & 48 & 1. & & n.d. & n.d. & n.d. & n.d. & n.d. \\
\hline $39-2,24-28$ & n.d. & 5520 & 318 & 375 & 1012 & 72100 & 44 & n.d. & 95 & n.d. & 55 & 1.5 & 63 & 29 & 55 & 2.0 & n.d. & n.d. & n.d. & n.d. & n.d. & n.d. \\
\hline $39-3,53-55$ & n.d. & 4860 & 240 & 363 & 885 & 66990 & 36 & n.d. & 132 & n.d. & 42 & 0.5 & 59 & & 51 & 1.0 & & n.d. & & & & \\
\hline $\begin{array}{l}40-1,14-17 \\
40-1,138-147\end{array}$ & n.d. & $\begin{array}{r}4740 \\
480\end{array}$ & 252 & $\begin{array}{l}419 \\
373\end{array}$ & $\begin{array}{l}885 \\
885\end{array}$ & $\begin{array}{l}64260 \\
64400\end{array}$ & 36 & n.d. & 130 & & 42 & 0.5 & $\begin{array}{l}65 \\
65\end{array}$ & $\begin{array}{l}25 \\
24\end{array}$ & $\begin{array}{l}51 \\
46\end{array}$ & 0.8 & $\begin{array}{l}\text { n.d. } \\
\text { n.d. }\end{array}$ & $\begin{array}{l}\text { n.d. } \\
\text { n.d. }\end{array}$ & $\begin{array}{l}\text { n.d. } \\
\text { n.d. }\end{array}$ & $\begin{array}{l}\text { n.d. } \\
\text { n.d. }\end{array}$ & $\begin{array}{l}\text { n.d. } \\
\text { n.d. }\end{array}$ & $\begin{array}{l}\text { n.d. } \\
\text { n.d. }\end{array}$ \\
\hline $40-3,80-83$ & $\begin{array}{l}\text { n.d. } \\
\text { n.d. }\end{array}$ & $\begin{array}{r}480 \\
4740\end{array}$ & $\begin{array}{l}248 \\
232\end{array}$ & $\begin{array}{l}373 \\
345\end{array}$ & 948 & $\begin{array}{l}64400 \\
64050\end{array}$ & $\begin{array}{l}37 \\
36\end{array}$ & $\begin{array}{l}\text { n.d. } \\
\text { n.d. }\end{array}$ & $\begin{array}{l}126 \\
120\end{array}$ & $\begin{array}{l}\text { n.d. } \\
\text { n.d. }\end{array}$ & $\begin{array}{l}45 \\
45\end{array}$ & $\begin{array}{l}0.0 \\
1.4\end{array}$ & $\begin{array}{l}65 \\
66\end{array}$ & $\begin{array}{l}24 \\
24\end{array}$ & $\begin{array}{l}46 \\
49\end{array}$ & 0. & n.d. & n.d. & n.d. & n.d. & n.d. & n.d. \\
\hline $40-4,67-72$ & 37.1 & 4740 & 244 & 359 & 885 & 64050 & 37 & n.d. & 117 & n.d. & 53 & 0.9 & 63 & 24 & 44 & 0.4 & n.d. & 0.04 & 0.97 & 0.71 & 0.47 & 1.24 \\
\hline $40-5,31-34$ & n.d. & 4740 & 245 & 187 & 948 & 65030 & 37 & n.d. & n.d. & n.d. & n.d. & 1.4 & 65 & 24 & 42 & 1.7 & n.d. & n.d. & n.d. & n.d. & n.d. & n.d. \\
\hline $41-1,128-130$ & n.d. & 4800 & 223 & 348 & 948 & 64680 & 37 & n.d. & 125 & n.d. & 42 & 2.1 & 57 & 24 & 48 & 0.4 & n.d. & n.d. & n.d. & n.d. & n.d. & n.d. \\
\hline 41 & & 4740 & 229 & 376 & 885 & 64400 & 36 & & 130 & & 43 & 0.7 & 65 & 21 & 50 & 1.2 & n.c & n.d. & n.d. & n.d. & n.d. & n.d. \\
\hline $41-2,136-138$ & n.d. & 4920 & 263 & 418 & 948 & 66710 & 40 & n.d. & 146 & n.d. & 44 & 1.1 & 66 & 25 & 55 & 0. & n.c & & n.d. & n.d. & n.d. & n.d. \\
\hline $42-2,40-44$ & n.d. & $\$ 100$ & 326 & 283 & 948 & 65100 & 50 & n.d. & 133 & n.d. & 52 & 0.0 & 61 & 27 & 50 & 1.3 & n.d. & n.d. & n.d. & n.d. & n.d. & n.d. \\
\hline $44-1,25-28$ & 43.3 & 5520 & 314 & 414 & 1012 & 68600 & 43 & 46.3 & 103 & 102 & 61 & 0.8 & 62 & 29 & 49 & 0.2 & n.d. & n.d. & 0.78 & 0.85 & 0.54 & 1.41 \\
\hline $44-2,93-96$ & d. & 5400 & 301 & 452 & 1012 & 71820 & 45 & n. & 114 & & 60 & & 59 & 29 & 47 & 1.0 & n. & & n.d. & n.c & n. & n.d. \\
\hline $45-1,85-88$ & n.d. & 5340 & 316 & 449 & 1012 & 68040 & 44 & n.d. & 112 & n.d. & 56 & 0.1 & 60 & 28 & 53 & 0.0 & n.d. & n.d. & 0.76 & n.d. & n.d. & n.d. \\
\hline $45-2,91-96$ & n.d. & 5700 & 307 & 376 & 1012 & 71890 & 43 & n.d. & 97 & n.d. & 62 & 1.5 & 67 & 28 & 55 & 0.1 & n.d. & n.d. & n.d. & n.d. & n.d. & n.d. \\
\hline $47-1,43-45$ & 42.1 & 5940 & 340 & 154 & 1075 & 77210 & 44 & 45.9 & 73 & 84 & 66 & 0.4 & 55 & 30 & 58 & 0.0 & n.d. & n.d. & 1.0 & 0.83 & 0.57 & 1.42 \\
\hline & & 5940 & 341 & 135 & 1138 & 79730 & 43 & & 74 & & 65 & & 55 & 30 & 51 & & n.c & n.d. & n.d. & & n.d. & n.d. \\
\hline $47-2,103-108$ & n.d. & 5700 & 357 & 176 & 948 & 74430 & 46 & n.d. & 79 & & 67 & 1.3 & 54 & 28 & 48 & 1.0 & n. & & n.d. & n. & & d. \\
\hline $47-3,24-27$ & n.d. & 5880 & 360 & 167 & 1012 & 71900 & 44 & n.d. & 79 & n.d. & 66 & & 4 & 29 & 46 & & n.d. & n.d. & 1.05 & n.d. & n.d. & n.d. \\
\hline $\begin{array}{l}48-2,80-84 \\
48-3,97-100\end{array}$ & $\begin{array}{l}\text { n.d. } \\
\text { n.d. }\end{array}$ & $\begin{array}{l}5880 \\
5160\end{array}$ & $\begin{array}{l}371 \\
331\end{array}$ & $\begin{array}{l}303 \\
313\end{array}$ & $\begin{array}{r}1075 \\
948\end{array}$ & $\begin{array}{l}75950 \\
67040\end{array}$ & $\begin{array}{l}46 \\
43\end{array}$ & $\begin{array}{l}\text { n.d. } \\
\text { n.d. }\end{array}$ & $\begin{array}{l}93 \\
96\end{array}$ & $\begin{array}{l}\text { n.d. } \\
\text { n.d. }\end{array}$ & $\begin{array}{l}70 \\
61\end{array}$ & $\begin{array}{l}1.5 \\
0.2\end{array}$ & $\begin{array}{l}54 \\
53\end{array}$ & $\begin{array}{l}30 \\
27\end{array}$ & $\begin{array}{l}56 \\
50\end{array}$ & $\begin{array}{l}1.0 \\
0.0\end{array}$ & $\begin{array}{l}\text { n.d. } \\
\text { n.d. }\end{array}$ & $\begin{array}{l}\text { n.d. } \\
\text { n.d. }\end{array}$ & $\begin{array}{l}\text { n.d. } \\
0.70\end{array}$ & $\begin{array}{l}\text { n.d. } \\
\text { n.d. }\end{array}$ & $\begin{array}{l}\text { n.d. } \\
\text { n.d. }\end{array}$ & $\begin{array}{l}\text { n.d. } \\
\text { n.d. }\end{array}$ \\
\hline $50-1,68-72$ & n.d. & 4980 & 262 & 382 & 948 & 65380 & 4 & & 10 & n.d. & 5 & 1 & 64 & 24 & 53 & & n.d. & n.d. & n.d. & n.d. & n.d. & n.d. \\
\hline $51-1$ & d. & 5040 & 270 & 387 & 948 & 6440 & 38 & n.c & 114 & & 50 & 0.2 & 63 & 2 & 5 & & & & n.d. & & $\mathrm{n}$. & n.d. \\
\hline $52-1,62-65$ & 37.0 & 5040 & 286 & 368 & 948 & 65100 & 37 & 40. & 108 & 123 & SC & 0.3 & 66 & 26 & 46 & 0.2 & n.d. & 0.02 & 0.91 & & 0.46 & 1.18 \\
\hline $52-2,55-60$ & n.d. & 5100 & 281 & 370 & 948 & 66220 & 36 & n.d & 105 & n. & 46 & 1.1 & 65 & 25 & 51 & & n.d. & n.d. & n.d. & n. & n. & \\
\hline $52-3,100-103$ & n.d. & 5040 & 288 & 368 & 948 & 64750 & 38 & n.c & 111 & n. & 45 & 0.6 & 64 & 25 & 49 & 1.5 & $\mathrm{n}$. & n.d. & n.d. & n.d. & n.d. & n.d. \\
\hline $52-4,63-66$ & n.d. & 5160 & 287 & 356 & 948 & 66430 & 39 & n.d. & 106 & n.d. & 53 & 1.2 & 64 & 26 & 49 & 0.4 & n.d. & n.d. & 0.75 & n.d. & n.d. & n.d. \\
\hline $54-1,16-18$ & 46.5 & 8160 & 353 & 271 & 1012 & 71540 & 43 & n.d. & 88 & n.d. & 60 & 0.0 & 106 & 35 & 97 & 3.1 & n.d. & n.d. & 3.60 & 1.25 & 0.73 & 2.55 \\
\hline $56-1,139-142$ & 46.5 & 8160 & 356 & 285 & 1075 & 73710 & 43 & n.d & 86 & & 6. & 0.4 & 107 & 37 & 106 & 3.0 & n.d. & n.d. & 3.20 & 1.24 & 0.72 & 2.54 \\
\hline $57-1,51-55$ & 45.8 & 8040 & 366 & 357 & 1012 & 69930 & 44 & n.d. & n.d. & n.d. & 62 & 0.3 & 107 & 37 & 105 & 4.0 & n.d. & n.d. & 2.90 & 1.22 & 0.72 & n.d. \\
\hline $57-2,26-30$ & n.d. & 5880 & 282 & 367 & 1012 & 69020 & 41 & n.d. & 117 & n.d. & 60 & t.t & 78 & 28 & 64 & 2.3 & n.d. & n.d. & 1.20 & n.d. & n.d. & n.d. \\
\hline $58-1,143-146$ & n.d. & 5400 & 322 & 265 & 1012 & 68110 & 38 & n.d. & 82 & n.d. & 57 & 1.6 & 53 & 27 & 58 & 0.9 & n.d. & n.d. & n.d. & n.d. & n.d. & n.d. \\
\hline $58-2,14-17$ & n.d. & 5460 & 319 & 289 & 1012 & 69440 & 38 & n.d. & & n.d. & 56 & 1.5 & 64 & 28 & 56 & 1.3 & n.d. & n.d. & n.d. & n.d. & n.d. & n.d. \\
\hline $59-1,46-48$ & n.d. & 6060 & 312 & 379 & 1012 & 69020 & 44 & n.d. & 119 & n.d. & 58 & 0.0 & 80 & 29 & 66 & 0.5 & n.d. & n.d. & 1.37 & n.d. & n.d. & n.d. \\
\hline & $\mathrm{n}$ & & 337 & 323 & 1075 & 7463 & 42 & n.d. & 92 & & 56 & 0.2 & 75 & 31 & & & n.d. & n.d. & n.d. & n.d. & n.d. & n.d. \\
\hline $61-1,112-115$ & n.d. & 6240 & 348 & 351 & 1138 & 77350 & 47 & n.d. & 122 & n.d. & 70 & 1.3 & 53 & 32 & 58 & 0.4 & n.d. & n.d. & n.d. & n.d. & n.d. & n.d. \\
\hline $63-2,103-105$ & & 6120 & 349 & 335 & 1075 & 74830 & 42 & n.d. & 118 & n.d. & 64 & 1.5 & 52 & 31 & 61 & 1.0 & & & & & & \\
\hline $64-2,107-109$ & 42.0 & 6960 & 363 & 150 & 1138 & 81690 & 43 & 44.8 & 59 & 73 & 71 & 0.4 & 57 & 35 & 72 & 0.8 & n.d. & n.d. & 1.20 & 0.92 & 0.64 & 1.83 \\
\hline $64-1,78-82$ & 41.8 & 5940 & 361 & 237 & 1075 & 76230 & 44 & 44.6 & 87 & 100 & 71 & 0.6 & 57 & 30 & 54 & 1.9 & n.d. & n.d. & 0.95 & 0.75 & 0.55 & 1.37 \\
\hline $70-1,56-60$ & 43.7 & 6540 & 364 & 172 & 1201 & 79870 & 45 & 46.4 & 71 & 81 & 64 & 1.5 & 59 & 33 & 65 & 1.5 & n.d. & n.d. & n.d. & 0.87 & 0.60 & 1.63 \\
\hline $505-25-1,26-28$ & 30.9 & 4260 & 224 & 605 & 1007 & 57050 & 39 & 40.8 & 202 & 222 & 52 & 0.6 & 81 & 19 & 42 & 1.1 & n.d. & 0.07 & 1.26 & 0.68 & 0.38 & 0.99 \\
\hline & n.d. & 4560 & 237 & 608 & 1084 & 57550 & 38 & n.d. & 173 & n.d. & 56 & 1.8 & 89 & 22 & 47 & 1.9 & n.d. & n.d. & n.d. & n.d. & n.d. & n.d. \\
\hline $505 \mathrm{~A}-1-1$ & 38.1 & & 272 & 449 & 1239 & & & 44.8 & 154 & 153 & 61 & 0.4 & 80 & & & & & & & 0.92 & 0.55 & 1.38 \\
\hline $2-1,51-55$ & 38.9 & 5700 & 266 & 436 & 1162 & 67830 & 41 & 44.1 & 148 & 166 & 67 & 1.1 & 74 & 28 & 57 & 0.9 & n.d. & & 1.49 & 0.83 & 0.74 & 1.43 \\
\hline $505 \mathrm{~B}-2-1,19-23$ & n.d. & 5880 & 271 & 430 & 1162 & 67130 & 44 & n.d. & 153 & n.d. & 61 & 0.4 & 75 & 29 & 56 & 1.0 & n.d. & n.d. & n.d. & n.d. & n.d. & n.d. \\
\hline & & & & & & & & & & & & & & & & & & & & & & \\
\hline $2-3,51-52$ & 38.1 & 5760 & 262 & 431 & 1394 & 67480 & 43 & 43. & 15 & 16 & 57 & 3.0 & 109 & 28 & 56 & & 0. & 0.14 & 1.22 & 0.8 & 0.4 & n.d. \\
\hline & & 5760 & 272 & 447 & 1084 & 6552 & 42 & 44 & & 17 & 58 & & 74 & 28 & 53 & & 0. & & 0.0 & 0.8 & 0. & 1.38 \\
\hline $3-1,76-79$ & 38.0 & 5700 & 270 & 455 & 1162 & 66780 & 41 & 44 & 157 & 149 & 60 & & 78 & 29 & 57 & 0.3 & 0.0 & 0.02 & 0.9 & 0.7 & 0. & 1.16 \\
\hline $3-2,102-105$ & 37.9 & 5700 & 267 & 437 & 1084 & 66570 & 44 & 44 & 168 & 174 & 59 & 0.6 & 77 & 27 & 56 & 1.2 & n.d. & & 1.5 & 0.84 & 0.54 & 1.50 \\
\hline $5-1,40-43$ & n.d. & 5820 & 261 & 437 & 1084 & 655 & 43 & n.d. & 167 & n.d. & 57 & 0.1 & 76 & 27 & 59 & 1. & n. & & n.d. & $\mathrm{n}$. & n. & n.d. \\
\hline $6-1,114-116$ & 39.8 & 5820 & 270 & 437 & 1162 & 66080 & 42 & 44.1 & 152 & 171 & 62 & 1.6 & 79 & 27 & 52 & i. & n.d. & & 1.38 & 0.91 & 0.72 & n.d. \\
\hline $6-2,20-23$ & 39.6 & 5820 & 263 & 441 & 1162 & 68040 & 43 & 44.4 & 165 & 161 & 62 & 0.5 & 75 & 27 & 58 & 1.3 & 0.01 & 0.02 & 1.37 & 0.92 & 0.65 & 1.49 \\
\hline
\end{tabular}

a Oxidized.

b Nonoxidized. 


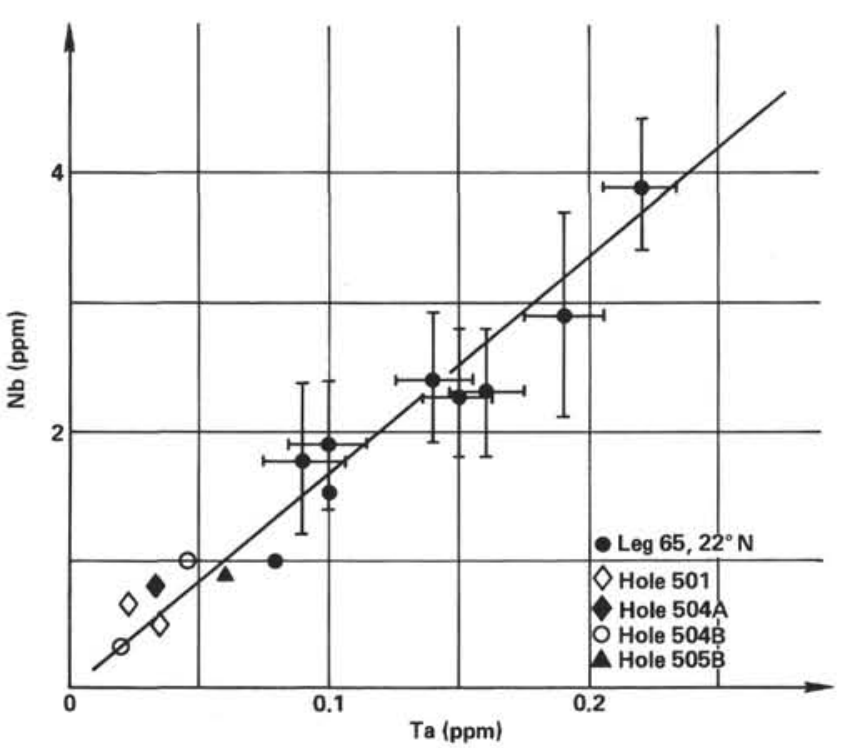

Figure 1. $\mathrm{Nb}$ versus Ta. Dots correspond to the different basaltic units encountered at $22^{\circ} \mathrm{N}$ on the East Pacific Rise during Leg 65. The two open circles are related to Hole 504B; the lower one corresponds to the averages $\mathrm{Ta} 0.02 \mathrm{ppm}$ and $\mathrm{Nb} 0.3 \mathrm{ppm}$; the upper one corresponds to the averages Ta $0.045 \mathrm{ppm}$ and $\mathrm{Nb} 1 \mathrm{ppm}$ (representing Samples 504B-16-3, 129-139 cm, 504B-17-2, 94-97 cm, and 504B-17-3, 22-24 cm.

value of the slope $\mathrm{Nb}=\mathrm{f}(\mathrm{Ta})$ derived from the leastsquares calculation is $\mathbf{1 6 . 4}$. It corresponds to the value found so far for oceanic basalts and is independent of the notion of enrichment or depletion of hygromagmatophile elements. On the other hand, all $\mathrm{Nb}, \mathrm{Ta}, \mathrm{La}$, and Th values, except four samples reported in Table 2 (Hole 504B) for Cores 18 and 19, probably lie within the $\Delta X$ values of these elements, and within twice their $d x$ values. In such cases, it is impossible to infer from Figure $1 \mathrm{a} \mathrm{Nb} / \mathrm{Ta}$ ratio for the low values encountered at Sites 501, 504, and 505. It is possible to say only that the values found are compatible with a $\mathrm{Nb} / \mathrm{Ta}$ ratio of 16 . There is no ambiguity for the larger concentrations encountered in Samples 504B-18-1, 84-88 cm, 504B-18-2, 98-102 cm, 504B-19-1, 73-77 cm, and 504B-19-2, 46-49 $\mathrm{cm}$, whose average $\mathrm{Nb} / \mathrm{Ta}$ ratio of 15.4 fits with the value $16 \pm 1$ of oceanic basalts.

$\mathrm{La}$ is plotted versus Ta in Figure 2, for $22^{\circ} \mathrm{N}$ samples of the East Pacific Rise (Leg 65), and for Sites 501, 504, and 505. Two values of the $\mathrm{La} / \mathrm{Ta}$ ratio have been found so far in the Atlantic: 9 and 19 (Bougault, Treuil, and Joron, 1979); only the value 19 has been found so far on the East Pacific Rise (Joron et al., 1980; Cambon et al., 1980). The same comments as for $\mathrm{Nb}$ and $\mathrm{Ta}$ can be made about the low La and Ta values at Sites 501, 504, and 505. It is difficult to give a $\mathrm{La} / \mathrm{Ta}$ ratio for these low concentrations, but it can be stated that these concentrations are compatible with the value 19 of the $\mathrm{La} / \mathrm{Ta}$ ratio. On the contrary, the four samples from Hole 504B (Table 2) represent the other value, 9 , of the $\mathrm{La} / \mathrm{Ta}$ ratio. This result is very important, because it is the first time that it is clearly established that basalts with low $\mathrm{La} / \mathrm{Ta}$ values of about 9 are found in the same hole as

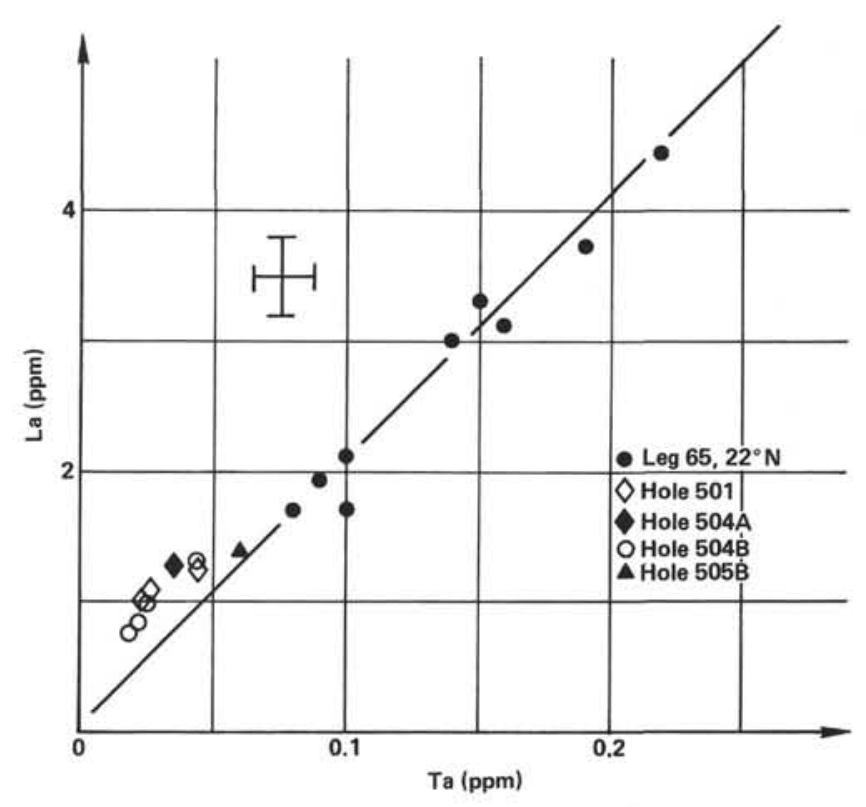

Figure 2. La versus Ta. (Compare Fig. 1.)

depleted basalts which probably have $\mathrm{La} / \mathrm{Ta}$ of approximately 18 .

It has recently been confirmed further that $\mathrm{Zr}$ and $\mathrm{Hf}$, as well as $\mathrm{Y}$ and $\mathrm{Tb}$, fractionate very little during magma genesis (Bougault, Treuil, and Joron, 1978). The values of $\mathrm{Zr} / \mathrm{Hf}$ and $\mathrm{Y} / \mathrm{Tb}$ ratios, 40 and 50 , respectively, are very close to the values already found for oceanic basalts.

There is a clear correlation between the concentrations of the various alkali metals. The observed variations are of the same order of magnitude as those observed at Hole $395 \mathrm{~A}$, at $22^{\circ} \mathrm{N}$ on the Mid-Atlantic Ridge (Bougault, Treuil, and Joron, 1979). These variations do not reflect primary variations, but appear to be the result of low-temperature alteration.

The high-partition-coefficient elements $\mathrm{Cr}$ and $\mathrm{Ni}$ show typical concentrations for tholeiites. The least-fractionated samples are two from Hole 505 (Table 2) which almost fulfill the theoretical requirements of being primary melts $(\mathrm{Cr}, 600 \mathrm{ppm}$; Ni, $250 \mathrm{ppm}$; Bougault, $1977 \mathrm{~b})$. The observed variations $(\mathrm{Cr}, 200-600 \mathrm{ppm}$; $\mathrm{Ni}$, $80-200 \mathrm{ppm}$ ) are probably due both to fractional crystallization and to the occurrence of some phenocrysts in some units.

\section{HYGROMAGMAPHILE ELEMENTS}

Several authors have long proposed different ways of presenting geochemical data, either on the basis of chemical properties of some elements, or as discriminants among different rock suites. Because of the available methods and equipment, authors use different elements to try to solve the same problems. For example, the normalized rare-earth-element diagram proposed by Coryell et al. (1963) and Masuda (1962) is one of the most popular. The triangular diagram for $\mathrm{Y}, \mathrm{Zr}$, and $\mathrm{Ti}$ of Pearce and Cann (1971) also is well known. Do Y, Zr, and $\mathrm{Ti}$ on the one hand, and rare-earth elements on the 
other, indicate different processes? If they are sensitive to the same processes, is it possible to find a unique way of presenting the data? Such attempts are common and lately have been based on the combination of rare earths and non-rare earths in an extended Coryell-Masuda plot. This plot uses concentrations normalized to chondrite abundances, and has the elements arranged according to their bulk behavior to the behavior of rare earths. The data presented in such a way have not always removed ambiguities, for several reasons: (1) some elements, such as alkali metals, are too sensitive to low-temperature alteration or sea-water contamination, which act to obscure initial abundances; (2) for other elements, such as $\mathrm{Zr}$, the normalizing concentrations (average chondrite concentrations) are not known with suitable precision (Schmitt et al., 1964; Ganapathy et al., 1976; Ehmann and Rebagay, 1970; Shima, 1979); (3) in such extended rare-earth-element diagrams, the dispersion of points along the "continuous" curve often is very large compared to the dispersion of the rare-earth elements themselves; this makes interpretation difficult, especially with respect to the evidence of possible anomalies, such as the Eu anomaly (Tarney, Wood, Varet, et al., 1979; Sun et al., 1979; Tarney, Wood, Saunders, et al., 1980; Morrison et al., 1980; Sun, 1980); (4) very few homogeneous data (rare-earth and non-rare-earth concentrations measured from the same samples) are available in the literature for samples which present different characteristics.

One of us (Bougault, 1980), has proposed a method for presentation of the data which is based on an extended Coryell-Masuda plot, for the purpose of comparative geochemistry. This is not in opposition to approaches such as that proposed by Gast (1968), Shaw (1970), Langmuir et al. (1977), or by ourselves (Bougault, Joron, and Treuil, 1979). First of all, alkali elements, $\mathrm{Sr}$, and $\mathrm{Ba}$ are not included in such a representation, because of alteration and contamination problems. Only the elements of "hygromagmaphile" character (such as rare earths) are considered; these elements have low "bulk" solid/liquid partition coefficients, and show an affinity for the liquid phase of the magma. It is meaningless to plot high-partition-coefficient elements such as $\mathrm{Ni}$ or $\mathrm{Cr}$ in such a diagram, because such elements behave during magmatic processes in different ways than hygromagmaphile elements. Such hygromagmaphile elements correspond to transition elements whose ions show a rare-gas electronic structure (except Group V). Instead of choosing single or average values as normalizing concentrations for non-rare earth elements from the chondritic concentrations proposed in the literature, the normalizing concentrations have been calculated by using an "inverse method," accounting for all of the homogeneous (analytical, obtained from the same samples) data of oceanic basalts obtained since Leg 37. Similarly, the position of non-rare-earth elements among rare earths has been determined using an inversion method. This classification of the elements on the basis of their hygromagmaphile character relies upon the continuous variation of the hygromagmaphile character of the rare earths.
The classification has been confirmed by a more theoretical approach, involving the incompatibility of these elements in a crystal structure, and their availability to form complexes in the liquid. The parameter $\phi$, which accounts for these two factors, involving ionic radius, $R$, and the ionic charge, $n$, is reported in Table 3 , together with the normalizing concentrations. These results have three implications: (1) the expression large ion lithophile element (LILE) has to be abandoned: Ta and Nb $(R=0.68 \AA)$ behave like La $(R=1.06 \AA)$. We propose replacement of this expression by "hygromagmaphile," to account for the affinity of the element for the liquid phase of the magma; (2) rare-earth and non-rareearth elements plot in an extended Coryell-Masuda diagram with the same precision; (3) anomalies, in addition to the widely known $\mathrm{Eu}$ anomaly, have been observed: there are possible Ti negative anomalies, possibly caused by opaque-mineral crystallization (El Azzou$\mathrm{zi}, 1981$ ), and there are anomalies in the abundance of $\mathrm{La}$ compared with $\mathrm{Nb}$ and $\mathrm{Ta}$, which have been interpreted so far in terms of mantle heterogeneity.

On the basis of the extended Coryell-Masuda plots, the data available for Sites 501, 504, and 505 can be divided into three categories. First, the category which corresponds to the majority of samples would plot in Figure 3 between the filled circles and filled diamonds. These basaltic units are more depleted in $\mathrm{Th}, \mathrm{Ta}, \mathrm{Nb}$, and $\mathrm{La}$ than any other rocks drilled during the DSDP project. The second category is represented by only three samples (Table 2; Hole 504B, 54-1 to 57-1), which are less depleted in $\mathrm{La}$ and $\mathrm{Nb}$ than those of category 1 . The third category is represented by four samples of Unit 5 of Hole 504B (Samples 504B-18-1, 84-85 cm to 504B-19-2, 46-49 cm). The normalized concentrations of hygromagmaphile elements present a flat distribution, even somewhat enriched in $\mathrm{Th}, \mathrm{La}, \mathrm{Nb}$. The major difference between these and samples of the two other categories lies in the fact that the $\mathrm{La}, \mathrm{Ta}$, and $\mathrm{Nb}$ normalized concentrations have the same value: this feature corresponds to the $\mathrm{La} / \mathrm{Ta}$ ratio equal to 9 , mentioned earlier. For category 2 , even if the Ta concentration is not available at the moment, from the $\mathrm{Nb}$ position, and using a $\mathrm{Nb} / \mathrm{Ta}$ ratio equal to 16 , the $\mathrm{La} / \mathrm{Ta}$ ratio of category 2 can be estimated to be close to 19 , based on Figure 3. For the first category, as already discussed in the preceding paragraph, because of the very low values, it is not possible to propose a value for the $\mathrm{La} / \mathrm{Ta}$ ratio, but the data and the position of the related points in Figure 3 are compatible with the value 19.

One important result of drilling the Costa Rica Rift lies in the La/Ta anomaly. Either the value 9 (normalized ratio: 1) or the value 19 (normalized ratio: 2 ) has been found so far at one site and for large areas of the sea floor. This bimodal distribution has heretofore been interpreted in terms of mantle domains, and it is very difficult to propose another interpretation at the moment. If the values of $\mathrm{La} / \mathrm{Ta}$ do reflect mantle heterogeneity, then the two values found in Hole 504B imply that the mantle can be locally heterogeneous. This result is clearly shown in Figure 3 by the relative position of $\mathrm{Nb}$ and Ta points compared to $\mathrm{La}$; either the open diamonds 


\section{J. ETOUBLEAU ET AL.}

Table 3. Classification and normalized concentrations of hygromagmaphile transition elements.

\begin{tabular}{cccccccccccccccccccccccc}
\hline Parameter & $\mathrm{Th}$ & $\mathrm{La}$ & $\mathrm{Ta}$ & $\mathrm{Nb}$ & $\mathrm{Ce}$ & $\mathrm{Pr}$ & $\mathrm{Nd}$ & $\mathrm{Zr}$ & $\mathrm{Hf}$ & $\mathrm{Sm}$ & $\mathrm{Ti}$ & $\mathrm{Eu}$ & $\mathrm{Gd}$ & $\mathrm{Tb}$ & $\mathrm{Y}$ & $\mathrm{Dy}$ & $\mathrm{Ho}$ & $\mathrm{Er}$ & $\mathrm{Tm}$ & $\mathrm{Yb}$ & $\mathrm{Lu}$ & $\mathrm{V}$ & $\mathrm{Sc}$ \\
\hline $\mathrm{Ch}$ & 0.028 & 0.32 & 0.031 & 0.53 & 0.85 & 0.112 & 0.60 & 5.13 & 0.128 & 0.19 & 460 & 0.07 & 0.25 & 0.047 & 2.16 & 0.32 & 0.07 & 0.21 & 0.03 & 0.20 & 0.033 & 22 & \\
$R$ & 0.99 & 1.06 & 0.68 & 0.69 & 1.03 & & 0.99 & 0.79 & 0.79 & 0.96 & 0.68 & 0.95 & 0.94 & 0.92 & 0.92 & 0.90 & & 0.88 & & 0.86 & 0.85 & 0.59 & 0.73 \\
$n$ & 4 & 3 & 5 & 5 & 3 & 3 & 3 & 4 & 4 & 3 & 4 & 3 & 3 & 3 & 3 & 3 & 3 & 3 & 3 & 3 & 3 & $(5)$ & 3 \\
$\phi$ & 8.34 & 7.35 & 7.25 & 7.35 & 6.90 & & 6.33 & 5.74 & 5.74 & 5.89 & 5.88 & 5.71 & 5.66 & 5.37 & 5.34 & 5.16 & & 4.93 & & 4.72 & 4.64 & $(9.23)$ & 4.22 \\
\hline
\end{tabular}

Note: $\mathrm{Ch}=$ chondrite-normalized concentrations; $R=$ ionic radius; $n=$ ionic charge or oxidation state; $\phi=$ computed hygromagmaphile character.

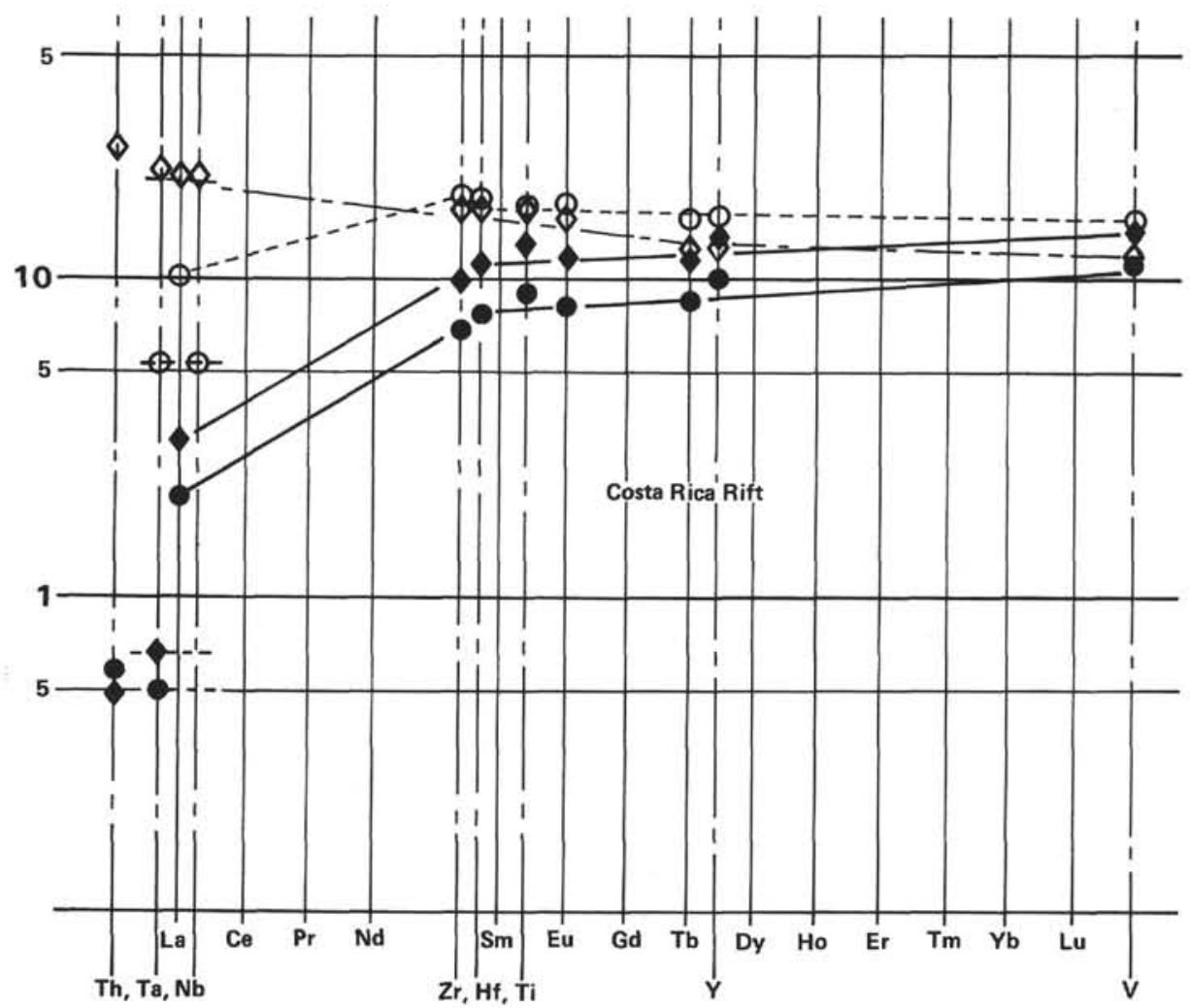

Figure 3. Costa Rica Rift, extended Coryell-Masuda plot. The examples chosen on this figure are from Hole 504B (Table 2). Filled circles: 504B-21-5 to 504B-22-2; filled diamonds, 504B-25-2, 96-99 cm; open circles: 504B-54-1 to 504B-57-1, 51-55 cm; open diamonds: 504B-54-1 to 504B-57-1, 51-55 cm. Because of low Ta and $\mathrm{Nb}$ values and related accuracies (see Fig. 1), the positions of filled diamonds and circles have to be associated with a large error for those two elements.

(with normalized $\mathrm{La} / \mathrm{Ta}$ or $\mathrm{Nb} / \mathrm{La}$ of 1 ) or $\mathrm{Nb}$ and/or Ta plot at a lower position than $\mathrm{La}$ (with normalized $\mathrm{La} / \mathrm{Ta}$ or $\mathrm{Nb} / \mathrm{La}$ of 2 ).

\section{CONCLUSIONS}

The basalts drilled from the Costa Rica Rift are more depleted in the most-hygromagmaphile elements (Th, $\mathrm{Ta}, \mathrm{Nb}$, and $\mathrm{La}$ ) than any other basalts drilled under the aegis of DSDP. Most of the concentrations of these elements are close to the precision limits of neutronactivation analysis and X-ray fluorescence. Despite the very low concentrations of $\mathrm{Ta}, \mathrm{Nb}$, and $\mathrm{La}$, it can be stated that these data are compatible with the value $16 \pm 1$ of the invariant ratio $\mathrm{Nb} / \mathrm{Ta}$, and the value 19 of the $\mathrm{La} / \mathrm{Ta}$. The two other invariant ratios, $\mathrm{Zr} / \mathrm{Hf}$ and $\mathrm{Y} / \mathrm{Tb}$, show the typical values 40 and 50 respectively.

Among more than 160 analyzed samples, only four samples present a flat or slightly enriched extended rare- earth pattern and a $\mathrm{La} / \mathrm{Ta}$ ratio equal to 9 ( 1 for the normalized ratio). Previously, we believed that these ratios characterized large mantle domains, based on the observation that large areas are characterized by one of the two $\mathrm{La} / \mathrm{Ta}$ (or $\mathrm{La} / \mathrm{Nb}$ ) ratios, but this finding (both ratios in the same hole) suggests that local mantle heterogeneity is likely.

\section{REFERENCES}

Bougault, H., 1977a. Major elements: analytical chemistry on board and preliminary results. In Aumento, F., Melson, W. G., et al., Init. Repts. DSDP, 37: Washington (U.S. Govt. Printing Office), 643-652.

1977b. Evidence de la cristallisation fractionnées au niveau d'une ride médio-océanique: $\mathrm{Co}, \mathrm{Ni}, \mathrm{Cr}, \mathrm{FAMOUS}$, Leg $37 \mathrm{du}$ D.S.D.P. Bull. Soc. Geol. France, 19:1207-1212.

1980. Contribution des eléments de transition à la compréhension de la genèse des basaltes océaniques; analyse des éléments traces dans les roches par spectrométrie de fluorescence $\mathrm{X}$ [thèse]. Université Paris VII. 
Bougault, H., Cambon, P., Corre, O., Joron, J. L., and Treuil, M., 1979. Evidence of variability of magmatic processes and upper mantle heterogeneity in the axial region of the mid-Atlantic Ridge near $22^{\circ}$ and $36^{\circ} \mathrm{N}$. Tectonophysics, 55:11-34.

Bougault, H., Cambon, P., and Toulhoat, H., 1977. X-ray spectrometric analysis of trace elements in rocks. Correction for instrumental interferences. X-Ray Spectrometry, 6:66-72.

Bougault, H., Joron, J. L., and Treuil, M., 1979. Atteration, fractional crystallization, partial melting, mantle properties from trace elements in basalts recovered in the North Atlantic. In Talwani, M., Harrison, C. G., and Hayes, D. E. (Eds.), Deep Drilling Results in the Atlantic Ocean: Ocean Crust: Washington (Am. Geophys. Union), 352-368.

Bougault, H., Treuil, M., and Joron, J. L., 1978. Trace elements in basalts from $23^{\circ} \mathrm{N}$ and $36^{\circ} \mathrm{N}$ in the Atlantic Ocean: fractional crystallization, partial melting and heterogeneity of the upper mantle. In Melson, W. G., Rabinowitz, P. D., et al., Init. Repts. DSDP, 45: Washington (U.S. Govt. Printing Office), 493-506.

Cambon, P., Joron, J. L., Bougault, H. and Treuil, M., 1980. Emperor Seamounts: trace elements in transitional tholeiites, alkali basalts and Hawaiites: mantle homogeneity or heterogeneity and magmatic processes. In Jackson, E. D., Koizumi, I., et al., Init. Repts. DSDP, 55: Washington (U.S. Govt. Printing Office), 585-598.

Coryell, C. D., Chase, J. W. and Winchester, J. W., 1963. A procedure for geochemical interpretation of terrestrial rare-earth abundance patterns. J. Geophys. Res., 68:559.

Ehmann, W. D., and Rebagay, T. V., 1970. Zirconium and hafnium in meteorites by activation analysis. Geochim. Cosmochim. Acta, 34: 649.

El Azzouzi, M., 1981. Géochimie comparée de quelques éléments hygromagmaphiles dans les roches volcaniques de contextes géodynamiques variés [thèse]. Université de Bretagne Occidentale.

Ganapathy, R., Papia G. M. and Grossman, L., 1976. The abundances of zirconium and hafnium in the solar system. Earth Planet. Sci. Lett., 29:302.

Gast, P. W., 1968. Trace element fractionation and the origin of tholeiitic and alkaline magma types. Geochim. Cosmochim. Acta, 32: 1057-1086.

Jaffrezic, H., Joron, J. L., and Treuil, M., 1977. Trace elements determination in rocks powder. A study of the precision for a given analytical procedure. Instrumental epithermal neutron activation. J. Radioanal. Chem., 39:185-188.

Joron, J. L., Briqueu, L., Bougault, H. and Treuil, M., 1980. East Pacific Rise, Galapagos spreading center and Siqueiros fracture zone, Deep Sea Drilling Project Leg 54; hygromagmaphile elements. A comparison with the North Atlantic. In Rosendahl, B. R., Hekinian, R., et al., Init. Repts. DSDP, 54: Washington (U.S. Govt. Printing Office), 725-735.

Langmuir, C. H., Bender, J. F., Bence, A. E., Hanson, G. N., and Taylor, S. R., 1977. Petrogenesis of basalts from the FAMOUS area: Mid-Atlantic Ridge. Earth Planet. Sci. Lett., 36:133-156.

Masuda, A., 1962. Regularities in variation of relative abundances of lanthanide elements and an attempt to analyze separation-index patterns of some minerals. J. Earth Sci. Nagoya Univ., 10: 173-187.

Melson, W. G., Rabinowitz, P. D., Natland, J. H., and Johnson, H. P., 1978. Cruise objects and major results: analytical procedures and explanatory notes. In Melson, W. G., Rabinowitz, P. D., et al., Init. Repts. DSDP, 45: Washington (U.S. Govt. Printing Office), 5-20.

Morrison, M. A., Thompson, R. N., Gibson, G. I. L., and Marriner, G. F., 1980. Lateral chemical heterogeneity in the Palaeocene upper mantle beneath the Scottish Hebrides. Philos. Trans. R. Soc. London, Ser. A, 297:229-244.

Pearce, J. A., and Cann, J. R., 1971. Ophiolite origin investigated by discriminant analysis using $\mathrm{Ti}, \mathrm{Zr}$ and $\mathrm{Y}$. Earth Planet. Sci. Lett., 12:339-349.

Schmitt, R. A., Bingham, E., and Chodos, A., 1964. Zirconium abundances in meteorites and implications to nucleosynthesis. Geochim. Cosmochim. Acta, 28:1961-1979.

Shaw, D. M., 1970. Trace element fractionation during anatexis. Geochim. Cosmochim. Acta, 34:237-243.

Shima, M., 1979. The abundances of titanium and hafnium in story meteorites. Geochim. Cosmochim. Acta, 43:353-362.

Sun, S. S., 1980. Lead isotopic study of young volcanic rocks from mid-ocean ridges, ocean islands and island arcs. Phil. Trans. Royal Soc. London, Ser. A, 297:409-445.

Sun, S. S., Nesbitt, R. W., and Sharaskin, A. Y., 1979. Geochemical characteristics of mid-ocean Ridge basalts, Earth Planet. Sci. Lett., 44:119-138.

Tarney, J., Wood, D. A., Saunders, A. D., Cann, J. R., and Varet J., 1980. Nature of mantle heterogeneity in the North Atlantic: evidence from deep sea drilling. Phil. Trans, Royal Soc. London, Ser. $A$, 207:179-202.

Tarney, J., Wood, D. A., Varet J., Saunders, A. D., and Cann, J. R., 1979. Nature of mantle heterogeneity in the North Atlantic: evidence from Leg 49 basalts. In Talwani, M., Harrison, C. G., and Hayes, D. E. (Eds.), Deep Drilling Results in the Atlantic Ocean: Ocean Crust: Washington (Am. Geophys. Union), pp. 285-301. 